WATPPHYS-TH01/09

\title{
Abelian Higgs Hair for Rotating and Charged Black Holes
}

\author{
A. M. Ghezelbash † and R. B. Mann ${ }^{\ddagger}$ \\ $\dagger, \ddagger$ Department of Physics, University of Waterloo, \\ Waterloo, Ontario N2L 3G1, CANADA \\ ${ }^{\dagger}$ Department of Physics, Alzahra University, \\ Tehran 19834, IRAN
}

October 22, 2018

\begin{abstract}
We study the problem of vortex solutions in the background of rotating black holes in both asymptotically flat and asymptoticlly anti de Sitter spacetimes. We demonstrate the Abelian Higgs field equations in the background of four dimensional Kerr, Kerr-AdS and Reissner-Nordstrom-AdS black holes have vortex line solutions. These solutions, which have axial symmetry, are generalization of the Nielsen-Olesen string. By numerically solving the field equations in each case, we find that these black holes can support an Abelian Higgs field as hair. This situation holds even in the extremal case, and no flux-expulsion occurs. We also compute the effect of the self gravity of the Abelian Higgs field show that the the vortex induces a deficit angle in the corresponding black hole metrics.
\end{abstract}

\footnotetext{
${ }^{1}$ EMail: amasoud@avatar.uwaterloo.ca

2 EMail: mann@avatar.uwaterloo.ca
} 


\section{Introduction}

The conjecture that the only long-range information associated with the endpoint of gravitational collapse is that of its total mass, angular momentum and electric charge is referred to as the no-hair conjecture of black holes [1]. Much work has been carried out over the years on this conjecture, either upholding it in certain instances (e.g. scalar fields [2]) or challenging it in others, such painting Yang-Mills, quantum hair [3] or Nielsen-Olesen vortices [4] on black holes. In fact the uniqueness of the classical no-hair theorems is a qualified uniqueness [5], incorporating additional criteria associated with stability, non trivial topology, and the possibility of field configurations on the horizon (referred to as 'dressing'), and one must be quite specific about what is meant by 'hair' 41. The study of Nielsen-Olesen vortices in the background of the charged black holes was done in [6], [7], [8] and [9].

Virtually all efforts in this area have been concerned with asymptotically flat spacetimes, and it is only recently that extensions to other types of asymptotia have been considered. Scalar fields minimally coupled to gravity cannot provide hair for asymptotically de Sitter black holes [10], but can do so if the spacetime is asymptotically anti de Sitter (AdS) [11]. A solution to the $S U(2)$ Einstein-Yang-Mills equations that describes a stable Yang-Mills hairy asymptotically AdS black hole has been shown to exist [3]. In a recent paper with Dehghani, we have shown that the $U(1)$ Higgs field equations have a vortex solution in both four dimensional AdS spacetime [12] and AdS-Schwarzschild backgrounds [13].

In this paper we extend our investigation of possible vortex hair for non-asymptotically flat black holes to include rotation and charge. Specifically, we seek numerical solutions of the AbelianHiggs field equations in the four dimensional rotating Kerr-AdS and Reissner-Nordstrom-AdS black hole backgrounds. Although an analytic or approximate solution to these equations appears to be intractable, we confirm by numerical calculation that Kerr-AdS and Reissner-NordstromAdS black holes could support a long range vortex (or cosmic string) as a form of stable hair. This is the first demonstration that rotating black holds can carry Abelian Higgs hair, for both the asymptotically flat and asymptotically AdS cases. We also show (to first order in the gravitational coupling) that the effect of the vortex on a rotating black hole is to create a deficit angle through the spacetime, analogous to its non-rotating counterpart. We consider the question of flux-expulsion by extremal black holes and find that in the case of extremal Kerr-AdS, Kerr and Reissner-Nordstrom-AdS black holes, an extremal horizon is indeed pierced by the vortex, and its flux is not expelled from the black holes. This not only confirms earlier computations of non-expulsion of vortex lines [8, 9] for the extremal Reissner-Nordstrom case, but also proves the non-expulsion phenomenon to the extremal rotating case in asymptotically AdS and asymptotically flat spacetimes. Finally, we consider the dependence of the characteristic vortex on the rotation parameter of the black hole and find that it is qualitatively the same its dependence on the magnitude of the cosmological constant: an increase in both causes a decrease in the thickness of the core.

In section two, we solve numerically the Abelian-Higgs equations in the Kerr-AdS background 
for different values of the cosmological constant and black hole rotation parameter. In section three, we consider the Abelian-Higgs equations in the limiting case of Kerr-AdS background with cosmological constant to be zero, yielding the vortex equation in the Kerr black hole background. In section four, we repeat this calculation for a Reissner-Nordstrom-AdS background for different values of the black hole charge. In the limiting case of Reissner-Nordstrom-AdS black hole with cosmological constant to be zero, yielding Reissner-Nordstrom black hole, our solution is in good agreement with the previous obtained results [9]. In section five, by an analytical discussion, we obtain the effect of the vortex self gravity on the Kerr black hole to the first order of gravitational constant. We establish that the effect of the vortex is to induce a deficit angle in the Kerr background and find the deficit angle in terms of vortex fields. Then in section six, by studying the behaviour of the string energy-momentum tensor, we find the effect of the vortex self gravity on the Kerr-AdS and Reissner-Nordstrom-AdS black hole backgrounds. We give some closing remarks in the final section.

\section{Abelian Higgs Vortex in Kerr-AdS Black Hole}

The Abelian Higgs Lagrangian is

$$
\mathcal{L}\left(\Phi, A_{\mu}\right)=-\frac{1}{2}\left(\mathcal{D}_{\mu} \Phi\right)^{\dagger} \mathcal{D}^{\mu} \Phi-\frac{1}{16 \pi} \mathcal{F}_{\mu \nu} \mathcal{F}^{\mu \nu}-\xi\left(\Phi^{\dagger} \Phi-\eta^{2}\right)^{2}
$$

where $\Phi$ is a complex scalar Klein-Gordon field, $\mathcal{F}_{\mu \nu}$ is the field strength of the electromagnetic field $A_{\mu}$ and $\mathcal{D}_{\mu}=\nabla_{\mu}+i e A_{\mu}$ in which $\nabla_{\mu}$ is the covariant derivative in a spacetime with metric $g_{\mu \nu}$. We employ Planck units $G=\hbar=c=1$ which implies that the Planck mass is equal to unity, and write the Kerr-AdS black hole metric in the analogue of Boyer-Lindquist coordinates

$$
d s^{2}=-\frac{\Delta_{r}}{\rho^{2}}\left(d t-\frac{a}{\Sigma} \sin ^{2} \theta d \varphi\right)^{2}+\frac{\rho^{2}}{\Delta_{r}} d r^{2}+\frac{\rho^{2}}{\Delta_{\theta}} d \theta^{2}+\frac{\Delta_{\theta} \sin ^{2} \theta}{\rho^{2}}\left(a d t-\frac{r^{2}+a^{2}}{\Sigma} d \varphi\right)^{2}
$$

where

$$
\begin{gathered}
\Delta_{r}=\left(r^{2}+a^{2}\right)\left(1+\frac{r^{2}}{l^{2}}\right)-2 m r \\
\Delta_{\theta}=1-\frac{a^{2} \cos ^{2} \theta}{l^{2}} \\
\Sigma=1-\frac{a^{2}}{l^{2}}
\end{gathered}
$$

and $\rho^{2}=r^{2}+a^{2} \cos ^{2} \theta$. The parameter $m$ is related to the mass of the black hole $M$ by

$$
M=\frac{m l}{\left(1-\frac{a^{2}}{l^{2}}\right)^{2}}
$$

and $a$ to the angular momentum $J$ by

$$
J=\frac{a m}{\left(1-\frac{a^{2}}{l^{2}}\right)^{2}}
$$


The cosmological constant $\Lambda$ is equal to $\frac{-3}{l^{2}}$. The metric (2) is valid only where $|a|<l$ and is singular where $|a|=l$. If $m>0, \Delta_{r}$ has at most two real roots and the event horizon is located at $r=r_{H}$, the largest real root of the equation $\Delta_{r}=0$. For some values of the parameters $a, m, l$, the two real roots coincide in which case the Kerr-AdS black hole is extremal. If roots are not real, then the spacetime described by the metric (2) is a naked singularity. As in the non-rotating case [13], we define the real fields $X\left(x^{\mu}\right), \omega\left(x^{\mu}\right), P_{\mu}\left(x^{\nu}\right)$ via the following equations

$$
\begin{aligned}
& \Phi\left(x^{\mu}\right)=\eta X\left(x^{\mu}\right) e^{i \omega\left(x^{\mu}\right)} \\
& A_{\mu}\left(x^{\nu}\right)=\frac{1}{e}\left(P_{\mu}\left(x^{\nu}\right)-\nabla_{\mu} \omega\left(x^{\mu}\right)\right)
\end{aligned}
$$

from which we can rewrite the Lagrangian (1) and the equations of motion in terms of these fields as

$$
\begin{gathered}
\mathcal{L}\left(X, P_{\mu}\right)=-\frac{\eta^{2}}{2}\left(\nabla_{\mu} X \nabla^{\mu} X+X^{2} P_{\mu} P^{\mu}\right)-\frac{1}{16 \pi e^{2}} F_{\mu \nu} F^{\mu \nu}-\xi \eta^{4}\left(X^{2}-1\right)^{2} \\
\nabla_{\mu} \nabla^{\mu} X-X P_{\mu} P^{\mu}-4 \xi \eta^{2} X\left(X^{2}-1\right)=0 \\
\nabla_{\mu} F^{\mu \nu}-4 \pi e^{2} \eta^{2} P^{\nu} X^{2}=0
\end{gathered}
$$

where $F^{\mu \nu}=\nabla^{\mu} P^{\nu}-\nabla^{\nu} P^{\mu}$ is the field strength of the corresponding gauge field $P^{\mu}$.

Provided the field $\omega$ is not single valued, the resultant solutions contain physical information and are referred to as vortex solutions [14]. The requirement that $\Phi$ be single-valued implies that the line integral of $\omega$ over any closed loop is $\pm 2 \pi n$ where $n$ is an integer. Through such a closed loop the flux of electromagnetic field $\Phi_{H}$ is quantized with quanta $2 \pi / e$.

We seek a vortex solution for the Abelian Higgs Lagrangian (6) in the background of Kerr-AdS black hole. This solution can be interpreted as a string piercing the black hole (2). Considering the static case of winding number $N$ with the gauge choice,

$$
P_{\mu}(r, \theta)=(0 ; 0,0, N P(r, \theta))
$$

and $X=X(r, \theta)$, we rescale

$$
\varkappa \rightarrow \frac{\varkappa}{\sqrt{\xi} \eta}
$$

where $\varkappa=r, l, m$, thereby obtaining

$$
\begin{gathered}
\frac{\left(r^{2}+l^{2}\right)\left(r^{2}+a^{2}\right)-2 m r l^{2}}{l^{2}\left(r^{2}+a^{2} \cos ^{2} \theta\right)} \frac{\partial^{2} X}{\partial r^{2}}+2 \frac{2 r^{3}+r\left(l^{2}+a^{2}\right)-m l^{2}}{l^{2}\left(r^{2}+a^{2} \cos ^{2} \theta\right)} \frac{\partial X}{\partial r}+\frac{l^{2}-a^{2} \cos ^{2} \theta}{l^{2}\left(r^{2}+a^{2} \cos ^{2} \theta\right)} \frac{\partial^{2} X}{\partial \theta^{2}}+\cot \theta \frac{l^{2}+2 a^{2}-3 a^{2} \cos ^{2} \theta}{l^{2}\left(r^{2}+a^{2} \cos ^{2} \theta\right)} \frac{\partial X}{\partial \theta} \\
+\frac{(a-l)^{2}(a+l)^{2}\left(r^{4}+\left(r^{2}+a^{2} \cos ^{2} \theta\right)\left(a^{2}+l^{2}\right)-a^{4} \cos ^{4} \theta+2 m r l^{2}\right)}{l^{2} \sin ^{2} \theta\left(r^{2}+a^{2} \cos ^{2} \theta\right)\left(a^{2} \cos ^{2} \theta-l^{2}\right)\left(r^{4}+r^{2}\left(a^{2}+l^{2}\right)+a^{2} l^{2}-2 m r l^{2}\right)} N^{2} X P^{2}-\frac{1}{2} X\left(X^{2}-1\right)=0 \\
-\frac{\left(r^{2}+l^{2}\right)\left(r^{2}+a^{2}\right)-2 m r l^{2}}{l^{2}\left(r^{2}+a^{2} \cos ^{2} \theta\right)} \frac{\partial^{2} P}{\partial r^{2}}-2 \frac{\left(a^{2}+r^{2}\right)\left(r^{5}+2 a^{2} \cos ^{2} \theta r^{3}+m l^{2} r^{2}+a^{4} \cos ^{4} \theta r-a^{2} m l^{2} \cos ^{2} \theta\right)}{l^{2}\left(r^{2}+a^{2} \cos ^{2} \theta\right)^{3}}+\frac{\partial P}{\partial r}+\frac{a^{2} \cos ^{2} \theta-l^{2}}{l^{2}\left(r^{2}+a^{2} \cos ^{2} \theta\right)} \frac{\partial^{2} P}{\partial \theta^{2}} \\
+\cot \theta \frac{r^{4}\left(l^{2}-2 a^{2}+a^{2} \cos ^{2} \theta\right)+2 a^{2} r^{2}\left(l^{2} \cos ^{2} \theta+a^{2} \cos ^{4} \theta-2 a^{2} \cos ^{2} \theta\right)+4 a^{2} m l^{2} \sin ^{2} \theta r+a^{4} \cos ^{2} \theta\left(l^{2} \cos ^{2} \theta+a^{2} \cos ^{4} \theta-2 a^{2}\right)}{l^{2}\left(r^{2}+a^{2} \cos ^{2} \theta\right)^{3}} \frac{\partial P}{\partial \theta} \\
+\alpha P X^{2}=0
\end{gathered}
$$


for the equations of motion (8), where $\alpha=\frac{4 \pi e^{2}}{\xi}$. The equations (10) and (11) in the special cases of $a=0$ and $a=m=0$ reduce to the equations of motion of the vortex in the Schwarzschild-AdS and AdS backgrounds respectively [13], 12].

We emphasize that even in the simplest case of an Abelian Higgs vortex in asymptotically flat spacetime ((10) and (11) in the limit $l \rightarrow \infty, a=0)$, no solution that is everywhere analytic has been found. Indeed, even for $m=0$ no exact analytic solutions are known for equations (10) and (11). Furthermore, no vortex solutions have ever been obtained for rotating black hole spacetimes. We now proceed with a numerical search for the existence of vortex solutions for the above coupled non linear partial differential equations.

First, we consider the thin string with winding number one, in which one can assume $m>>1$. Thicker vortices and larger winding numbers will be discussed later in this section. Employing the ansatz

$$
P(r, \theta)=P(R), \quad X(r, \theta)=X(R)
$$

where $R=r \sin \theta$, we obtain the following equations:

$$
\begin{gathered}
-\frac{a^{2}\left(R^{4}+r^{4}-3 r^{2} R^{2}-R^{2} l^{2}\right)-r^{4}\left(l^{2}+R^{2}\right)+2 r m l^{2} R^{2}}{l^{2}\left(r^{4}+a^{2}\left(r^{2}-R^{2}\right)\right)} \frac{d^{2} X}{d R^{2}}-\frac{a^{2}\left(r^{4}-7 r^{2} R^{2}+4 R^{4}\right)-r^{4}\left(4 R^{2}+l^{2}\right)+2 R^{2} m l^{2} r}{R l^{2}\left(r^{4}+a^{2}\left(r^{2}-R^{2}\right)\right)} \frac{d X}{d R} \\
-\frac{1}{2}\left(X^{3}-X\right)+f(r, \theta) N^{2} X P^{2}=0
\end{gathered}
$$

$\frac{r^{2}\left(a^{2} \cos ^{4} \theta-l^{2}\right)-\sin ^{2} \theta\left(r^{4}+a^{2} r^{2}-2 m r l^{2}+a^{2} l^{2}\right)}{l^{2}\left(r^{2}+a^{2} \cos ^{2} \theta\right)} \frac{d^{2} P}{d R^{2}}+\frac{g(r, \theta)}{l^{2} \sin \theta\left(r^{2}+a^{2} \cos ^{2} \theta\right)^{3}} \frac{d P}{d R}-\alpha P X^{2}=0$

where the functions $f(r, \theta)$ and $g(r, \theta)$ are given by,

$$
\begin{gathered}
f(r, \theta)=\frac{r^{2}(a-l)^{2}(a+l)^{2}\left(a^{4} R^{2} r^{2}-a^{4} R^{4}+a^{2} r^{6}+a^{2} r^{4} l^{2}-a^{2} R^{2} l^{2} r^{2}-2 r^{5} m l^{2}+r^{8}+l^{2} r^{6}\right)}{R^{2} l^{2}\left\{r^{4}+a^{2}\left(r^{2}-R^{2}\right)\right\}\left\{r^{2}\left(a^{2}-l^{2}\right)-a^{2} R^{2}\right\}\left\{\left(r^{2}+l^{2}\right)\left(r^{2}+a^{2}\right)-2 m r l^{2}\right\}} \\
\left\{\begin{array}{c}
g(r, \theta)=-2 \sin ^{2} \theta r^{7}+\left(6 \cos ^{4} \theta a^{2}+l^{2}-5 a^{2} \cos ^{2} \theta-2 a^{2}\right) r^{5}-2 m l^{2} \sin ^{2} \theta r^{4}+ \\
\left\{-4 a^{4} \cos ^{2} \theta\left(1+\cos ^{2} \theta\right)+2 a^{2} l^{2} \cos ^{2} \theta+6 a^{4} \cos ^{6} \theta\right\} r^{3}-2 a^{2} m l^{2}\left(1+3 \cos ^{4} \theta-4 \cos ^{2} \theta\right) r^{2} \\
+a^{4} \cos ^{4} \theta\left(l^{2}-2 a^{2}+2 a^{2} \cos ^{4} \theta-a^{2} \cos ^{2} \theta\right)+2 a^{4} m l^{2} \sin ^{2} \theta \cos ^{2} \theta
\end{array}\right.
\end{gathered}
$$

When $a=0$, the vortex solutions of the Abelian Higgs equations in flat spacetime (without a black hole) satisfy the $l \rightarrow \infty$ limit of equations up to errors which are proportional to $\frac{m R^{2}}{r^{3}} \approx$ $\frac{m}{r^{3}}$ [12. These errors are very tiny far from the black hole horizon, whereas near the horizon $r \approx r_{H}=2 m$, they are of the order of $\frac{1}{m^{2}}$, which is negligible for large mass black holes. This suggests that a string vortex solution could be painted to the horizon of a Schwarzschild black hole, and numerical calculations [4 have indeed shown the existence of vortex solutions of the Abelian Higgs equations in this background. 
The situation is somewhat different for finite $l$. For $a=0$ (and $l \neq 0$ ) we have shown in a previous paper [12] that the Abelian Higgs equations of motion in the background of Anti-deSitter spacetime ( (13) and (14) in the limit of $a=0$ and $m=0$ ) have vortex solutions (denoted by $X_{0}$ and $P_{0}$ ) with core radius $R \approx O(1)$. The functions $X_{0}$ and $P_{0}$ satisfy eqs. (13) and (14) up to errors which are proportional to $\frac{m R^{2}}{r^{3}} \approx \frac{m}{r^{3}}$. Although these errors go to zero far from the black hole, for a large mass black hole, $r \approx r_{H} \approx m^{1 / 3}$, the term $\frac{m}{r^{3}}$ is at least of the order of unity, and so the possibility of obtaining a string vortex solution for finite $l$ in this background is unclear.

By numerically solving eqs. (13,14) in the $a=0$ case we have shown that vortex solutions exist on, near and far from the horizon of the AdS-Schwarzschild black hole for various winding numbers and different values of $l$ [13]. As in the asymptotically flat case the results indicate that increasing the winding number yields a greater vortex thickness. Furthermore as $l$ decreases the black hole becomes completely covered by a vortex of decreasingly large winding number. Also, for a vortex with definite winding number, the string core decreases with decreasing $l$, but the ratio of string core to the size of the black hole horizon increases. The $X$ and $P$ fields less rapidly approach their respective maximum and minimum values at larger angles as $l$ decreases.

For finite values of the rotation parameter $a$, equations (13) and (14) which are equivalent to equations (10) and (11) are considerably more complicated than in the $a=0$ case. To obtain numerical solutions of (10) and (11) outside the black hole horizon we must first select appropriate boundary conditions. At large distances from the horizon physical considerations motivate a clear choice: we demand that the $a \neq 0$ solutions approach the solutions of the vortex equations in pure AdS spacetime given in ref. [12]. This means that we demand $X \rightarrow 1$ and $P \rightarrow 0$ as $R$ goes to infinity. On the symmetry axis of the string and beyond the radius of horizon $r_{H}$, i.e. $\theta=0$ and $\theta=\pi$, we take $X \rightarrow 0$ and $P \rightarrow 1$. Finally, on the horizon, we initially take $X=0$ and $P=1$.

We then employ a polar grid of points $\left(r_{i}, \theta_{j}\right)$, where $r$ goes from $r_{H}$ to some large value of $r$ ( $r_{\infty}$ ) which is much greater than $r_{H}$ and $\theta$ runs from 0 to $\pi$. We use the finite difference method and rewrite the non linear partial differential equation (10) and (11) as

$$
\begin{gathered}
A_{i j} X_{i+1, j}+B_{i j} X_{i-1, j}+C_{i j} X_{i, j+1}+D_{i j} X_{i, j-1}+E_{i j} X_{i, j}=F_{i j} \\
A_{i j}^{\prime} P_{i+1, j}+B_{i j}^{\prime} P_{i-1, j}+C_{i j}^{\prime} P_{i, j+1}+D_{i j}^{\prime} P_{i, j-1}+E_{i j}^{\prime} P_{i, j}=F_{i j}^{\prime}
\end{gathered}
$$

where $X_{i j}=X\left(r_{i}, \theta_{j}\right)$ and $P_{i j}=P\left(r_{i}, \theta_{j}\right)$. For the interior grid points and horizon grid points, the coefficients $A_{i j}, \ldots, F_{i j}^{\prime}$ can be straightforwardly determined from the corresponding continued differential equations (10) and (11). The form of the coefficients is somewhat complicated, and we so relegate them to an appendix.

Using the well known successive overrelaxation method [15 for the above mentioned finite difference equations, we obtain the values of $X$ and $P$ fields inside the grid, which we denote them by $X^{(1)}$ and $P^{(1)}$. Then by calculating the $r$-gradients of $X$ and $P$ just outside the horizon and iterating the finite difference equations on the horizon, we get the new values of $X$ and $P$ fields on the horizon points. Then these new values of $X$ and $P$ fields are used as the new boundary condition on the horizon for the next step in obtaining the values of $X$ and $P$ fields inside the grid which could be denoted by $X^{(2)}$ and $P^{(2)}$. Repeating this procedure, the value of the each field in the $(n+1)$-th iteration is related to the $n$-th iteration by 


$$
\begin{aligned}
& X_{i j}^{(n+1)}=X_{i j}^{(n)}-\omega \frac{\zeta_{i j}^{(n)}}{E_{i j}^{(n)}} \\
& P_{i j}^{(n+1)}=P_{i j}^{(n)}-\omega \frac{\varsigma_{i j}^{(n)}}{E_{i j}^{\prime(n)}}
\end{aligned}
$$

where the residual matrices $\zeta_{i j}^{(n)}$ and $\varsigma_{i j}^{(n)}$ are the differences between the left and right hand sides of the equations (17) and (18) respectively, evaluated in the $n$-th iteration and $\omega$ is the overrelaxation parameter. The iteration is performed many times to some value $n=K$, such that $\sum_{i, j}\left|X_{i j}^{(K)}-X_{i j}^{(K-1)}\right|<\varepsilon$ and $\sum_{i, j}\left|P_{i j}^{(K)}-P_{i j}^{(K-1)}\right|<\varepsilon$ for a given error $\varepsilon$. It is a matter of trial and error to find the value of $\omega$ that yields the most rapid convergence.

Some typical results of this calculation are displayed in figures (1), (2) and (4),(5), (6) for different values of $l=5$ and $l=10$, respectively. In the first case, we consider both $a=0$ and $a=3$ and in the second case, we consider $a=0,5,8$. For $l=5$, the black hole mass is taken to be the constant value $m=10$ whereas for $l=10$ the mass is taken to be $m=20$. In $l=5$, the horizon is located in $r_{H}=6.89$ for $a=0$ and $r_{H}=6.30$ for $a=3$. Also, for the case of $l=10$, the horizon for different values of $a=0,5,8$ are $r_{H}=13.79,12.98,11.57$ respectively. For these two values of $l$, the black holes are non-extremal for all values of $a$. The diagrams (1) and (4) when $a=0$ are exactly the same as the results of [13] for the AdS-Schwarzschild black hole. We notice that by increasing the rotation parameter $a$ from 0 to 3 , the string core decreases slightly in the $l=5$ Kerr-AdS black hole. Figure (3) shows explicitly the string core decreasing as the rotation parameter increases.

The physical reason for the core size decreasing with increasing rotation parameter $a$ is due to a decrease in the horizon radius $r_{H}$. This quantity is given by the root of equation (3) and decreases slowly as the parameter $a$ increases from 0 to its maximum value. On the other hand, since we know from previous work that the string thickness compared to horizon radius drastically changes by changing unbounded physical quantities like the winding number or black hole mass, we expect that string core changes a little with changing the bounded black hole parameters like the rotation parameter $a$. Since the effect of increasing $l$ (when $a=0$ ) is the same as the effect of decreasing $a$ on the value of the horizon size $r_{H}$, we expect commensurate changes in the horizon size. Indeed this is what we find: the core thickness increases with decreasing parameter $a$, analogous to what happens when the value of $l$ is increased for $a=0$.

Figure (17) illustrates for $l=10$ a similar decrease of the string core as $a$ increases from 0 to 5 and then to 8, confirming again our expectation. Our numerical results agree with the above statement relating the horizon radius and different physical quantities of the black hole. Similar calculations for the string with larger winding numbers in a Kerr-AdS black hole with definite parameters $l, a$, show that increasing the winding number yields a greater vortex thickness.

For the extremal Kerr-AdS black hole with cosmological parameters $l=10$, mass $m=11$ and rotation parameter $a=7.939195$ we have calculated the vortex fields. In this case the horizon 
radius is located at $r_{H}=5.11015$. We find that the behaviour of the vortex $X$ and $P$ fields are the same as the non-extremal cases. The contours of the $X$ and $P$ fields attach to the horizon in the same way they attach to the horizon of non extremal black holes presented in the figures 1,2,4, 5,6. Since the results are so similar to the non-extremal cases we do not present them here. The crucial point is that in the extremal case, no flux-expulsion is occurred in the thin vortex configuration.

\section{Abelian Higgs Vortex in a Kerr Black Hole}

We consider here a special case of the preceding section, namely the Abelian Higgs vortex in a Kerr black hole background. The Kerr metric is a special case of (2) in the limit $l \rightarrow \infty$. The metric is given by

$$
d s^{2}=-\frac{\Delta}{\rho^{2}}\left(d t-a \sin ^{2} \theta d \varphi\right)^{2}+\frac{\rho^{2}}{\Delta} d r^{2}+\rho^{2} d \theta^{2}+\frac{\sin ^{2} \theta}{\rho^{2}}\left(\left(r^{2}+a^{2}\right) d \varphi-a d t\right)^{2}
$$

where $\Delta=r^{2}-2 m r+a^{2}$. The horizon is located in $r_{H}=m+\sqrt{m^{2}-a^{2}}$. The equations of motion for the $X$ and $P$ fields can be obtained from the equations (10) and (11) in the limit of $l \rightarrow \infty$. They are,

$$
\begin{aligned}
& \frac{r^{2}+a^{2}-2 m r}{r^{2}+a^{2} \cos ^{2} \theta} \frac{\partial^{2} X(r, \theta)}{\partial r^{2}}+2 \frac{r-m}{r^{2}+a^{2} \cos ^{2} \theta} \frac{\partial X(r, \theta)}{\partial r}+\frac{1}{r^{2}+a^{2} \cos ^{2} \theta} \frac{\partial^{2} X(r, \theta)}{\partial \theta^{2}}+\cot \theta \frac{1}{r^{2}+a^{2} \cos ^{2} \theta} \frac{\partial X(r, \theta)}{\partial \theta} \\
& -\frac{r^{2}+a^{2} \cos ^{2} \theta-2 m r}{\sin ^{2} \theta\left(r^{2}+a^{2}-2 m r\right)\left(r^{2}+a^{2} \cos ^{2} \theta\right)} N^{2} X(r, \theta) P(r, \theta)^{2}-\frac{1}{2} X(r, \theta)\left(X(r, \theta)^{2}-1\right)=0 \\
& -\frac{r^{2}+a^{2}-2 m r}{r^{2}+a^{2} \cos ^{2} \theta} \frac{\partial^{2} P(r, \theta)}{\partial r^{2}}-2 m \frac{\left(a^{2}+r^{2}\right)\left(r^{2}-a^{2} \cos ^{2} \theta\right)}{\left(r^{2}+a^{2} \cos ^{2} \theta\right)^{3}} \frac{\partial P(r, \theta)}{\partial r}-\frac{1}{r^{2}+a^{2} \cos ^{2} \theta} \frac{\partial^{2} P(r, \theta)}{\partial \theta^{2}} \\
& +\cot \theta\left(\frac{1}{r^{2}+a^{2} \cos ^{2} \theta}+4 \frac{r m a^{2} \sin ^{2} \theta}{\left(r^{2}+a^{2} \cos ^{2} \theta\right)^{3}}\right) \frac{\partial P(r, \theta)}{\partial \theta}+\alpha P(r, \theta) X^{2}(r, \theta)=0
\end{aligned}
$$

We solved the differential equations (22) and (23) numerically, using the same approach employed in solving (10) and (11). Some typical results of the calculation are displayed in figures (8), (9) and (10). In these calculations, we consider a string with unit winding number in the background

(21) with $a=0,5,9$ along the extremal case $a=10$. Throughout the black hole mass is taken to be $m=10$, and so the horizon is respectively located at $r_{H}=20,18.66,14.36$, with $r_{H}=m=10$ in the extremal case.

The figures in (8), when $a=0$ are exactly the same as the results of the Schwarzschild black hole [1], [12]. We note that in changing of the rotation parameter from a value of 0 (corresponding to a Schwarzschild black hole) to the extremal value of 10 , both the $X$ and $P$ contours undergo only a small change. In fact, as the diagrams in figure (11) show, we observe a change on the $X=0.9$ and $P=0.1$ contours. By increasing the rotation parameter, both the $X$ and $P$ contours decrease a little. Similar arguments regarding the decrease of the horizon of a Kerr-AdS black hole with increasing rotation parameter also apply to the Kerr black hole, which explains the decrease of the string fields $X$ and $P$ in figure (11) with increasing $a$. In the extremal case we find that an extremal horizon is indeed pierced by a thin string, so the vortex flux is not expelled from the black hole. 


\section{Abelian Higgs Vortex in Reissner-Nordstrom-AdS and Reissner-Nordstrom black holes}

In this section, we consider the Abelian Higgs vortex Lagrangian (1) in the background of a charged black hole. The background metric is given by,

$$
d s^{2}=-\left(1-\frac{2 m}{r}+\frac{Q^{2}}{r^{2}}+\frac{r^{2}}{l^{2}}\right) d t^{2}+\frac{1}{1-\frac{2 m}{r}+\frac{Q^{2}}{r^{2}}+\frac{r^{2}}{l^{2}}} d r^{2}+r^{2}\left(d \theta^{2}+\sin ^{2} \theta d \varphi^{2}\right)
$$

$Q$ is the total charge of black hole which measured by a far observer located at $r \gg 2 M, Q$, and the black hole horizon $r_{H}$ is located at the largest real root of the equation $r^{4}+l^{2} r^{2}-2 m l^{2} r+Q^{2} l^{2}=0$. In the special case of $l \rightarrow \infty$, the horizon is located at $r_{H}=m+\sqrt{m^{2}-Q^{2}}$. The Abelian Higgs Lagrangian is as the same as (1) with the real fields $X\left(x^{\mu}\right), \omega\left(x^{\mu}\right), P_{\mu}\left(x^{\nu}\right)$ again given by the relations (5), whose equations of motion are (6) and (7). The equations of motion derived from the Lagrangian for the $X(r, \theta)$ and $P(r, \theta)$ fields after rescaling of coordinates in the background (24) are

$$
\begin{aligned}
& \left(1-\frac{2 m}{r}+\frac{Q^{2}}{r^{2}}+\frac{r^{2}}{l^{2}}\right) \frac{\partial^{2} X(r, \theta)}{\partial r^{2}}+\frac{2}{r}\left(1-\frac{m}{r}+\frac{2 r^{2}}{l^{2}}\right) \frac{\partial X(r, \theta)}{\partial r}+\frac{1}{r^{2}} \frac{\partial^{2} X(r, \theta)}{\partial \theta^{2}}+\frac{1}{r^{2}} \frac{\partial X(r, \theta)}{\partial \theta} \cot \theta-\frac{1}{2}\left(X^{3}(r, \theta)\right. \\
& -X(r, \theta))-N^{2} \frac{X(r, \theta) P^{2}(r, \theta)}{r^{2} \sin ^{2} \theta}=0 \\
& \quad\left(1-\frac{2 m}{r}+\frac{Q^{2}}{r^{2}}+\frac{r^{2}}{l^{2}}\right) \frac{\partial^{2} P(r, \theta)}{\partial r^{2}}+\frac{2}{r}\left(\frac{m}{r}+\frac{r^{2}}{l^{2}}-\frac{Q^{2}}{r^{2}}\right) \frac{\partial P(r, \theta)}{\partial r}+\frac{1}{r^{2}} \frac{\partial^{2} P(r, \theta)}{\partial \theta^{2}}-\frac{\cot \theta}{r^{2}} \frac{\partial P(r, \theta)}{\partial \theta} \\
& -\alpha P(r, \theta) X^{2}(r, \theta)=0
\end{aligned}
$$

We note that in the special case $Q=0$, the equations of motion (25) and (26) reduce to the equations of motion of the vortex in the background of AdS-Schwarzschild background studied in [13]. Also we note that in the special case of $l \rightarrow \infty$, the above mentioned equations reduce to the equations of motion in the background of the Reissner-Nordstrom black hole discussed in [6] and [0].

We consider the static case of a string solution with winding number one. Using the overrelaxation method described in section two, we solve numerically the equations of motion. A typical result for the string fields in the background of the Reissner-Nordstrom-AdS black hole with $l=5$ and $Q=5$ is presented in figure (12). As figure (13) shows, by increasing the parameter $l$ from 3 to $\infty$, the string core changes a little. Such a tiny increase of string core also has been observed in the case of AdS-Schwarzschild black hole by increasing the parameter $l$ [13]. In the special case of $l \rightarrow \infty$, a typical solution of (25) and (26) for a Reissner-Nordstrom black hole with $m=10$ and $Q=5$ is shown in figure (14). In figure (15), the $X=0.9$ and $P=0.1$ contours are plotted for three different values of the charge parameter $Q=0,5,10$. The last case corresponds to extremal case $Q=m=10$. So, we observe that despite the smaller horizon radius for a black hole with more charge, the string core does not change drastically for the charged black holes for a wide range of the charge parameter $0 \leq Q \leq 10$. In the extremal case we find that an extremal horizon is indeed pierced by a thin string, confirming earlier computations carried out in references [8] and [9], and that the vortex flux is not expelled from the black hole. In these calculations, we have used a $600 \times 700$ grid in the $(r, \theta)$ directions, a much finer resolution than employed in previous studies [6, 7, 8, 9]. Note that there are no values of $Q$ or $m$ for which the black hole becomes extremal in the asymptotically AdS case. 


\section{Vortex Self Gravity and Kerr Black Holes}

In this section, we study the effect of the vortex on the Kerr black hole. The Kerr metric (21) can be written in the axisymmetric Weyl form as follows [16]:

$$
d s^{2}=-e^{2 U_{0}}\left(d t+A_{0} d \varphi\right)^{2}+e^{2\left(K_{0}-U_{0}\right)}\left(d \varrho^{2}+d z^{2}\right)+e^{-2 U_{0}} \varrho^{2} d \varphi^{2}
$$

where the functions $A_{0}, U_{0}, K_{0}$ are independent of $t, \varphi$ and are given by,

$$
\begin{gathered}
A_{0}=\operatorname{am} \frac{\left(4 \sigma^{2}-\left(r_{+}-r_{-}\right)^{2}\right)\left(2 m+r_{+}+r_{-}\right)}{\sigma^{2}\left[\left(r_{+}+r_{-}\right)^{2}-4 m^{2}\right]+a^{2}\left(r_{+}-r_{-}\right)^{2}} \\
U_{0}=\frac{1}{2} \ln \left\{\frac{\sigma^{2}\left[\left(r_{+}+r_{-}\right)^{2}-4 m^{2}\right]+a^{2}\left(r_{+}-r_{-}\right)^{2}}{\sigma^{2}\left(r_{+}+r_{-}+2 m\right)^{2}+a^{2}\left(r_{+}-r_{-}\right)^{2}}\right\} \\
K_{0}=\frac{1}{2} \ln \left\{\frac{\sigma^{2}\left[\left(r_{+}+r_{-}\right)^{2}-4 m^{2}\right]+a^{2}\left(r_{+}-r_{-}\right)^{2}}{4 \sigma^{2} r_{+} r_{-}}\right\}
\end{gathered}
$$

The quantities $r_{+}, r_{-}$and $\sigma$ are given by

$$
\begin{gathered}
r_{ \pm}=\sqrt{\varrho^{2}+(z \pm \sigma)^{2}} \\
\sigma=\sqrt{m^{2}-a^{2}}
\end{gathered}
$$

The transformation between the spherical coordinates in (21) and Weyl coordinates in (27) is given by,

$$
\begin{gathered}
\varrho=\sqrt{\Delta} \sin \theta=\sqrt{r^{2}-2 m r+a^{2}} \sin \theta \\
z=(r-m) \cos \theta
\end{gathered}
$$

These Weyl coordinates are useful for studying the back reaction of the vortex on the Kerr black hole.

In order to get the gravitational effect of the vortex on the Kerr black hole, we consider a general static axisymmetric metric of the form

$$
d s^{2}=-e^{2 U}(d t+A d \varphi)^{2}+e^{2(K-U)}\left(d \varrho^{2}+d z^{2}\right)+e^{-2 U} \xi^{2} d \varphi^{2}
$$

where $A, U, K$ and $\xi$ are dependent on the Weyl coordinates $\varrho$ and $z$. The Einstein equations become

$$
\begin{gathered}
\frac{\partial^{2} \xi}{\partial \varrho^{2}}+\frac{\partial^{2} \xi}{\partial z^{2}}=\varepsilon\left(T_{z}^{z}+T_{\varrho}^{\varrho}\right) \sqrt{-g} \\
e^{4 U}\left\{\left(\frac{\partial A}{\partial \varrho}\right)^{2}+\left(\frac{\partial A}{\partial z}\right)^{2}\right\} \xi+2\left(\frac{\partial^{2} U}{\partial \varrho^{2}}+\frac{\partial^{2} U}{\partial z^{2}}\right) \xi \\
+2 \frac{\partial U}{\partial z} \frac{\partial \xi}{\partial z}+2 \frac{\partial U}{\partial \varrho} \frac{\partial \xi}{\partial \varrho}+e^{4 U} A \xi\left(\frac{\partial^{2} A}{\partial \varrho^{2}}+\frac{\partial^{2} A}{\partial z^{2}}\right) \\
+3 e^{4 U} A\left(\frac{\partial A}{\partial \varrho} \frac{\partial \xi}{\partial \varrho}+\frac{\partial A}{\partial z} \frac{\partial \xi}{\partial z}\right)+4 e^{4 U}\left\{A \xi\left(\frac{\partial A}{\partial \varrho} \frac{\partial U}{\partial \varrho}+\frac{\partial A}{\partial z} \frac{\partial U}{\partial z}\right)\right. \\
\left.+A^{2}\left(\frac{\partial A}{\partial \varrho} \frac{\partial \xi}{\partial \varrho}+\frac{\partial A}{\partial z} \frac{\partial \xi}{\partial z}\right)\right\}+e^{4 U} A^{2}\left(\frac{\partial^{2} \xi}{\partial \varrho^{2}}+\frac{\partial^{2} \xi}{\partial z^{2}}\right)=-\varepsilon\left(T_{t}^{t}-T_{\varphi}^{\varphi}+T_{z}^{z}+T_{\varrho}^{\varrho}\right) \sqrt{-g}
\end{gathered}
$$




$$
\begin{gathered}
e^{4 U} \frac{\partial A}{\partial \varrho} \frac{\partial A}{\partial z} \xi^{2}+e^{4 U} \frac{\partial \xi}{\partial \varrho} \frac{\partial \xi}{\partial z} A^{2}+e^{4 U} A \xi\left(\frac{\partial A}{\partial \varrho} \frac{\partial \xi}{\partial z}+\frac{\partial A}{\partial z} \frac{\partial \xi}{\partial \varrho}\right) \\
-2 \xi \frac{\partial^{2} \xi}{\partial \varrho \partial z}-4 \xi^{2} \frac{\partial U}{\partial \varrho} \frac{\partial U}{\partial z}+2 \xi\left(\frac{\partial \xi}{\partial \varrho} \frac{\partial K}{\partial z}+\frac{\partial \xi}{\partial z} \frac{\partial K}{\partial \varrho}\right)=2 \varepsilon \xi\left(T \frac{\varrho}{z}\right) \sqrt{-g} \\
4 \xi^{2}\left\{\left(\frac{\partial U}{\partial \varrho}\right)^{2}+\left(\frac{\partial U}{\partial z}\right)^{2}\right\}+4 e^{4 U}\left(\frac{\partial A}{\partial \varrho} \frac{\partial \xi}{\partial \varrho}+\frac{\partial A}{\partial z} \frac{\partial \xi}{\partial z}\right) A \xi-e^{4 U}\left\{\left(\frac{\partial \xi}{\partial \varrho}\right)^{2}+\left(\frac{\partial \xi}{\partial z}\right)^{2}\right\} A^{2} \\
+4\left(\frac{\partial^{2} K}{\partial \varrho^{2}}+\frac{\partial^{2} K}{\partial z^{2}}\right) \xi^{2}+8 e^{4 U}\left(\frac{\partial A}{\partial \varrho} \frac{\partial U}{\partial \varrho}+\frac{\partial A}{\partial z} \frac{\partial U}{\partial z}\right) A \xi^{2} \\
+2 e^{4 U}\left(\frac{\partial^{2} A}{\partial \varrho^{2}}+\frac{\partial^{2} A}{\partial z^{2}}\right) A \xi^{2}+8 e^{4 U} \xi A^{2}\left(\frac{\partial U}{\partial \varrho} \frac{\partial \xi}{\partial \varrho}+\frac{\partial U}{\partial z} \frac{\partial \xi}{\partial z}\right)+2 e^{4 U} \xi A^{2}\left(\frac{\partial^{2} \xi}{\partial \varrho^{2}}+\frac{\partial^{2} \xi}{\partial z^{2}}\right) \\
+e^{4 U} \xi^{2}\left\{\left(\frac{\partial A}{\partial \varrho}\right)^{2}+\left(\frac{\partial A}{\partial z}\right)^{2}\right\} \\
=4 \varepsilon \xi(T \varphi) \sqrt{-g}
\end{gathered}
$$

where $\varepsilon=8 \pi \eta^{2}$ and $g$ is the determinant of the metric (31). The non-vanishing energy-momentum components are given by

$$
\begin{aligned}
& T_{t}^{t}=2\left(X^{2}-1\right)^{2}-\frac{e^{2 U} X^{2} P^{2}}{\xi^{2}}-e^{2(U-K)}\left\{\left(\frac{\partial X}{\partial \varrho}\right)^{2}+\left(\frac{\partial X}{\partial z}\right)^{2}\right\}+e^{2(2 U-K)} \frac{1}{\xi^{2}}\left\{\left(\frac{\partial P}{\partial \varrho}\right)^{2}+\left(\frac{\partial P}{\partial z}\right)^{2}\right\} \\
& T_{\varrho}^{\varrho}=2\left(X^{2}-1\right)^{2}-\frac{e^{2 U} X^{2} P^{2}}{\xi^{2}}+e^{2(U-K)}\left\{\left(\frac{\partial X}{\partial \varrho}\right)^{2}-\left(\frac{\partial X}{\partial z}\right)^{2}\right\}-e^{2(2 U-K)} \frac{1}{\xi^{2}}\left\{\left(\frac{\partial P}{\partial \varrho}\right)^{2}-\left(\frac{\partial P}{\partial z}\right)^{2}\right\} \\
& T_{z}^{z}=2\left(X^{2}-1\right)^{2}-\frac{e^{2 U} X^{2} P^{2}}{\xi^{2}}-e^{2(U-K)}\left\{\left(\frac{\partial X}{\partial \varrho}\right)^{2}-\left(\frac{\partial X}{\partial z}\right)^{2}\right\}+e^{2(2 U-K)} \frac{1}{\xi^{2}}\left\{\left(\frac{\partial P}{\partial \varrho}\right)^{2}-\left(\frac{\partial P}{\partial z}\right)^{2}\right\} \\
& T_{\varphi}^{\varphi}=2\left(X^{2}-1\right)^{2}+\frac{e^{2 U} X^{2} P^{2}}{\xi^{2}}-e^{2(U-K)}\left\{\left(\frac{\partial X}{\partial \varrho}\right)^{2}+\left(\frac{\partial X}{\partial z}\right)^{2}\right\}-e^{2(2 U-K)} \frac{1}{\xi^{2}}\left\{\left(\frac{\partial P}{\partial \varrho}\right)^{2}+\left(\frac{\partial P}{\partial z}\right)^{2}\right\} \\
& T_{z}^{\varrho}=2 e^{2(U-K)} \frac{\partial X}{\partial \varrho} \frac{\partial X}{\partial z}-2 e^{2(2 U-K)} \frac{1}{\xi^{2}} \frac{\partial P}{\partial \varrho} \frac{\partial P}{\partial z}
\end{aligned}
$$

Writing $U=U_{0}+\varepsilon U_{1}, K=K_{0}+\varepsilon K_{1}, A=A_{0}+\varepsilon A_{1}, \xi=\xi_{0}+\varepsilon \xi_{1}$, where $\xi_{0}=\varrho$, we can solve the equations (32)-(35) to first order in $\varepsilon$. To order zero in $\varepsilon$, the background metric is give by (27) and the string fields by $X_{0}(R)$ and $P_{0}(R)$. So to first order in $\varepsilon$, equation (32) becomes

$$
\frac{\partial^{2} \xi_{1}}{\partial \varrho^{2}}+\frac{\partial^{2} \xi_{1}}{\partial z^{2}}=e^{2\left(K_{0}-U_{0}\right)} \varrho\left(T_{z}^{z}+T_{\varrho}^{\varrho}\right)_{0}
$$

which can be solved by assuming the form $\xi_{1}=\varrho \zeta(R)$. Here, we take $R=\varrho e^{-U_{0}}$. Then function $\zeta(R)$ satisfies

$$
\frac{d^{2} \zeta}{d R^{2}}+\frac{2}{R} \frac{d \zeta}{d R}=\left(T_{z}^{z}+T_{\varrho}^{\varrho}\right)_{0}
$$

which can be solved to give

$$
\zeta(R)=\int \frac{\int R^{2}\left(T_{z}^{z}+T_{\varrho}^{\varrho}\right)_{0} d R}{R^{2}} d R=\int R\left(T_{z}^{z}+T_{\varrho}^{\varrho}\right)_{0} d R-\frac{1}{R} \int R^{2}\left(T_{z}^{z}+T_{\varrho}^{\varrho}\right)_{0} d R
$$

Since outside the vortex core the string fields $X_{0}(R)$ and $P_{0}(R)$ rapidly approach constant values, the integrals in (39) go correspondingly to the respective constant values $\tau_{1}$ and $\tau_{2}$. This gives $\xi=\rho\left(1+\varepsilon \tau_{1}\right)$, and the solutions of the other coupled equations (33)-(35) are found to be $U=U_{0}+\varepsilon v, \quad K=K_{0}+2 \varepsilon v$ and $A=A_{0}\left(1+\varepsilon \tau_{1}\right) e^{-2 \varepsilon v} \quad$ where $v=\frac{1}{2} \int \frac{\int R\left(T_{t}^{t}-T_{\varphi}^{\varphi}+T_{z}^{z}+T_{\varrho}^{\varrho}\right)_{0} d R}{R} d R$. Using these quantities in (31) and rescaling the coordinates $t, \varrho, z$ and function $A_{0}$, by $\widehat{t}=e^{\varepsilon v} t, \widehat{\varrho}=$ $e^{\varepsilon v} \varrho, \widehat{z}=e^{\varepsilon v} z$ and $\widehat{A}_{0}=e^{\varepsilon v} A_{0}$, we get finally,

$$
d s^{2}=-e^{2 U_{0}}\left\{d \widehat{t}+\widehat{A}_{0}\left(1+\varepsilon \tau_{1}\right) e^{-2 \varepsilon v} d \varphi\right\}^{2}+e^{2\left(K_{0}-U_{0}\right)}\left(d \widehat{\varrho}^{2}+d \widehat{z}^{2}\right)+e^{-2 U_{0}} \widehat{\varrho}^{2}\left(1+\varepsilon \tau_{1}\right)^{2} e^{-4 \varepsilon v} d \varphi^{2}
$$


which describes the Kerr metric with a deficit angle, since the angle $\widetilde{\varphi} \equiv\left(1+\varepsilon \tau_{1}\right) e^{-2 \varepsilon v} \varphi$ belongs to the interval $\left[0,2 \pi\left\{1+\varepsilon\left(\tau_{1}-2 v\right)\right\}\right]$.

\section{Vortex Self Gravity on the Kerr-AdS and Reissner-Nordstrom- AdS Black Holes}

We now first consider the effect of the vortex on the Kerr-AdS black hole. As we have seen in [13], this is a formidable problem even for the simpler cases of the effect of the vortex on the AdS-Schwarzschild or Schwarzschild black hole backgrounds.

For the AdS-Schwarzschild black hole, it has been shown that the components of the energymomentum tensor rapidly go to zero outside the core string, leading to a situation similar to that of pure AdS spacetime. A full study of vortex self gravity in pure AdS spacetime was carried out in ref. [13. We assume for the present case that the thickness of the vortex is much smaller than all other relevant length scales and that the gravitational effects of the string are weak enough so that the linearized Einstein-Abelian Higgs differential equations are applicable. So, we consider a thin string with the winding number $N=1$ in the Kerr-AdS background with $l=5$. The analysis for other values of $l$ is similar. The rescaled diagonal components of the energy-momentum tensor are

$$
\begin{aligned}
& T_{\mu \mu}=f_{\mu \mu}^{(r)}\left(\frac{\partial X}{\partial r}\right)^{2}+g_{\mu \mu}^{(r)}\left(\frac{\partial P}{\partial r}\right)^{2}+f_{\mu \mu}^{(\theta)}\left(\frac{\partial X}{\partial \theta}\right)^{2}+g_{\mu \mu}^{(\theta)}\left(\frac{\partial P}{\partial \theta}\right)^{2} \\
& +h_{1 \mu \mu}\left(X^{2}-1\right)^{2}+h_{2 \mu \mu} X^{2} P^{2}
\end{aligned}
$$

where the functions $f_{\mu \mu}^{(r)}, f_{\mu \mu}^{(\theta)}, g_{\mu \mu}^{(r)}, g_{\mu \mu}^{(\theta)}, h_{1 \mu \mu}$ and $h_{2 \mu \mu}$ are complicated functions of the coordinates $(r, \theta)$. Their functional forms are presented in the appendix. In the figure (16) the behaviour of energy-momentum tensor components for a fixed value of $z$ is shown. We have checked that the behaviour of the components other $z$ directions is similar.

It is clear from these figures that the components of the energy-momentum tensor rapidly go to zero outside the core of the vortex, rendering the situation similar to that of AdS-Schwarzschild spacetime. Performing the same calculation as for pure AdS spacetime described in detail in [13], we obtain the following metric for the Kerr-AdS spacetime incorporating the effect of the vortex

$$
d s^{2}=-\frac{\Delta_{r}}{\rho^{2}}\left(d t-\frac{a \beta}{\Sigma} \sin ^{2} \theta d \varphi\right)^{2}+\frac{\rho^{2}}{\Delta_{r}} d r^{2}+\frac{\rho^{2}}{\Delta_{\theta}} d \theta^{2}+\frac{\Delta_{\theta} \sin ^{2} \theta}{\rho^{2}}\left(a d t-\beta \frac{r^{2}+a^{2}}{\Sigma} d \varphi\right)^{2}
$$

which $\beta$ is a constant dependent on the different parameters of the black hole. The above metric describes a Kerr-AdS metric with a deficit angle. Also if we take the limiting case of $l \rightarrow \infty$, we get the following Kerr spacetime incorporated the effect of the string on it,

$$
d s^{2}=-\frac{\Delta}{\rho^{2}}\left(d t-a \gamma \sin ^{2} \theta d \varphi\right)^{2}+\frac{\rho^{2}}{\Delta} d r^{2}+\rho^{2} d \theta^{2}+\frac{\sin ^{2} \theta}{\rho^{2}}\left(\gamma\left(r^{2}+a^{2}\right) d \varphi-a d t\right)^{2}
$$

in which $\gamma$ is another constant that also depends on the different parameters of the Kerr black hole as noted above in (40). 
So, using a physical Lagrangian based model, we have established that the presence of the cosmic string induces a deficit angle in the Kerr-AdS and Kerr black holes metric. In the case of charged Reissner-Nordstrom-AdS black hole, the energy-momentum tensor also goes rapidly to zero outside the core string, so the above arguments are still applicable: the effect of the vortex on the background (24) simply multiplies the angle coordinate $\varphi$ by a constant, inducing a deficit angle in the Reissner-Nordstrom-AdS black hole spacetime.

\section{Conclusion}

The effect of a vortex on pure AdS spacetime is to create a deficit angle in the metric in the thin vortex approximation. We have extended this result to the charged and stationary cases, establishing numerically that Abelian Higgs vortices of finite thickness can pierce the Kerr, KerrAdS, and Reissner-Nordstrom-AdS black hole horizons. These solutions could thus be interpreted as stable Abelian hair for these black holes.

We have obtained numerical solutions for various cosmological constants and rotation parameter for a string with winding number one. Our solutions in the limit $a \rightarrow 0$ coincide with the known solutions in the AdS-Schwarzschild spacetime. We found that by increasing the value of rotation parameter, the string core decreases. Inclusion of the self gravity of the vortex in the Kerr-AdS background metric was shown to induce a deficit angle in this metric. We extended these results to obtained numerical solutions for a string with winding number one in the background of a Reissner-Nordstrom-AdS black hole for different values of the parameters.

We also considered extremal black holes in all three situations. We found by numerical integration that the string core remains nearly the same as that of a Schwarzschild black hole with the same mass. We observed that in the extremal case the string can pierce the charged/rotating black hole horizon and no flux expulsion occurs, an effect noted for charged black holes in the asymptotically flat case [8, 9]. Our results establish that flux-expulsion does not take place for extremal black holes, and that generically a cosmic vortex will pierce both rotating and charged black holes, with the stress-energy of the vortex inducing a deficit angle in these black hole metrics.

Other interesting issues concern the development of a holographic description of the vortex solution in these spacetimes. The holographic description of a vortex in was given by a discontinuity in the logarithmic derivative of a scalar-two point function in a CFT formulated on a spacetime with deficit angle. It is reasonable to conjecture that our extensions of vortex solutions to charged and rotating cases will yield a similar effect, but with the CFT at a finite temperature due to the presence of the black hole event horizon. Work on these problems is in progress.

\section{Appendix}

Here we present the coefficients $A_{i j}, \ldots, F_{i j}^{\prime}$ appear in the equations (17) and (18). Let us rename the coefficients of the $\frac{\partial^{2} X(r, \theta)}{\partial r^{2}}, \frac{\partial X(r, \theta)}{\partial r}, \frac{\partial^{2} X(r, \theta)}{\partial \theta^{2}}, \frac{\partial X(r, \theta)}{\partial \theta}$ and $X(r, \theta)$ (fifth term) appearing in the equation (10) by $X_{r r}, X_{r}, X_{\theta \theta}, X_{\theta}, \widetilde{X}$ respectively. Also, we rename the coefficients of the $\frac{\partial^{2} P(r, \theta)}{\partial r^{2}}, \frac{\partial P(r, \theta)}{\partial r}, \frac{\partial^{2} P(r, \theta)}{\partial \theta^{2}}, \frac{\partial P(r, \theta)}{\partial \theta}$ appearing in the equation (11) by $P_{r r}, P_{r}, P_{\theta \theta}, P_{\theta}$ respectively. Then 
the coefficients $A_{i j}, \ldots, F_{i j}$ inside the grid points are given by the following relations,

$$
\begin{gathered}
A_{i j}=\left\{\frac{X_{r r}}{(\Delta r)^{2}}+\frac{X_{r}}{2 \Delta r}\right\}_{r=r_{i}, \theta=\theta_{j}} \\
B_{i j}=\left\{\frac{X_{r r}}{(\Delta r)^{2}}-\frac{X_{r}}{2 \Delta r}\right\}_{r=r_{i}, \theta=\theta_{j}} \\
C_{i j}=\left\{\frac{X_{\theta \theta}}{(\Delta \theta)^{2}}+\frac{X_{\theta}}{2 \Delta \theta}\right\}_{r=r_{i}, \theta=\theta_{j}} \\
D_{i j}=\left\{\frac{X_{\theta \theta}}{(\Delta \theta)^{2}}-\frac{X_{\theta}}{2 \Delta \theta}\right\}_{r=r_{i}, \theta=\theta_{j}} \\
E_{i j}=\left\{-2\left(\frac{X_{r r}}{(\Delta r)^{2}}+\frac{X_{\theta \theta}}{(\Delta \theta)^{2}}\right)+\widetilde{X}\right\}_{r=r_{i}, \theta=\theta_{j}} \\
F_{i j}=-\frac{1}{2} X_{i j}\left(X_{i j}^{2}-1\right)
\end{gathered}
$$

The coefficients $A_{i j}^{\prime}, \ldots, D_{i j}^{\prime}$ have the similar form as $A_{i j}, \ldots, D_{i j}$ with the replacements $X_{r r}, X_{r}, X_{\theta \theta}, X_{\theta}$ by $P_{r r}, P_{r}, P_{\theta \theta}, P_{\theta}$ and

$$
E_{i j}^{\prime}=-2\left\{\frac{X_{r r}}{(\Delta r)^{2}}+\frac{X_{\theta \theta}}{(\Delta \theta)^{2}}\right\}_{r=r_{i}, \theta=\theta_{j}}+\alpha X_{i j}^{2}
$$

The coefficient $F_{i j}^{\prime}$ is equal to zero.

Here, we present also some of the functional form of the functions $f_{\mu \mu}^{(r)}, f_{\mu \mu}^{(\theta)}, g_{\mu \mu}^{(r)}, g_{\mu \mu}^{(\theta)}, h_{1 \mu \mu}$ and $h_{2 \mu \mu}$ which was appeared in the formula (41).

For example, we have

$$
\begin{gathered}
f_{t t}^{(r)}=\frac{\left(r^{2} l^{2}+r^{4}+a^{2} l^{2}+r^{2} a^{2}-2 m r l^{2}\right)\left(r^{2} l^{2}+r^{4}+r^{2} a^{2}-2 m r l^{2}+a^{2} l^{2} \cos ^{2} \theta+a^{4} \cos ^{2} \theta-a^{4} \cos ^{4} \theta\right)}{2 l^{4}\left(a^{2} \cos ^{2} \theta+r^{2}\right)^{2}} \\
f_{t t}^{(\theta)}=\frac{\left(a^{2} \cos ^{2} \theta-l^{2}\right)\left(-r^{2} l^{2}-r^{4}-r^{2} a^{2}+2 m r l^{2}-a^{2} l^{2} \cos ^{2} \theta-a^{4} \cos ^{2} \theta+a^{4} \cos ^{4} \theta\right)}{2 l^{4}\left(a^{2} \cos ^{2} \theta+r^{2}\right)^{2}} \\
g_{t t}^{(r)}=\frac{\left(r^{2} l^{2}+r^{4}+r^{2} a^{2}-2 m r l^{2}+a^{2} l^{2} \cos ^{2} \theta+a^{4} \cos ^{2} \theta-a^{4} \cos ^{4} \theta\right)^{2}\left(a^{2}-l^{2}\right)^{2}}{2 \alpha l^{6}\left(a^{2} \cos ^{2} \theta+r^{2}\right)^{3} \sin ^{2} \theta\left(l^{2}-a^{2} \cos ^{2} \theta\right)} \\
g_{t t}^{(\theta)}=\frac{\left(r^{2} l^{2}+r^{4}+r^{2} a^{2}-2 m r l^{2}+a^{2} l^{2} \cos ^{2} \theta+a^{4} \cos ^{2} \theta-a^{4} \cos ^{4} \theta\right)^{2}\left(a^{2}-l^{2}\right)^{2}}{2 \alpha l^{6}\left(a^{2} \cos ^{2} \theta+r^{2}\right)^{3} \sin ^{2} \theta\left(r^{2} l^{2}+r^{4}+a^{2} l^{2}+r^{2} a^{2}-2 m r l^{2}\right)} \\
h_{1 t t}=\frac{\left(r^{2} l^{2}+r^{4}+r^{2} a^{2}-2 m r l^{2}+a^{2} l^{2} \cos ^{2} \theta+a^{4} \cos ^{2} \theta \sin ^{2} \theta\right)}{l^{2}\left(a^{2} \cos ^{2} \theta+r^{2}\right)} \\
h_{2 t t}=\frac{\left(r^{2} l^{2}+r^{4}+r^{2} a^{2}-2 m r l^{2}+a^{2} l^{2} \cos ^{2} \theta+a^{4} \cos ^{2} \theta \sin ^{2} \theta\right)\left(a^{2}-l^{2}\right)^{2}}{2 l^{4}\left(a^{2} \cos ^{2} \theta-l^{2}\right) \sin ^{2} \theta\left(a^{2} \cos ^{2} \theta+r^{2}\right)^{2}\left(r^{2} l^{2}+r^{4}+a^{2} l^{2}+r^{2} a^{2}-2 m r l^{2}\right)}
\end{gathered}
$$

Other functions have similar complicated structures; we shall not present them here.

\section{Acknowledgments}

This work was supported by the Natural Sciences and Engineering Research Council of Canada.

\section{References}

[1] R. Ruffini and J.A. Wheeler. Phys. Today 24, 30 (1971).

[2] D. Sudarsky, Class. Quant. Grav. 12, 579 (1995).

[3] E. Winstanley, Class. Quant. Grav. 16, 1963 (1999).

[4] A. Achucarro, R. Gregory, K. Kuijken, Phys. Rev. D52, 5729 (1995). 

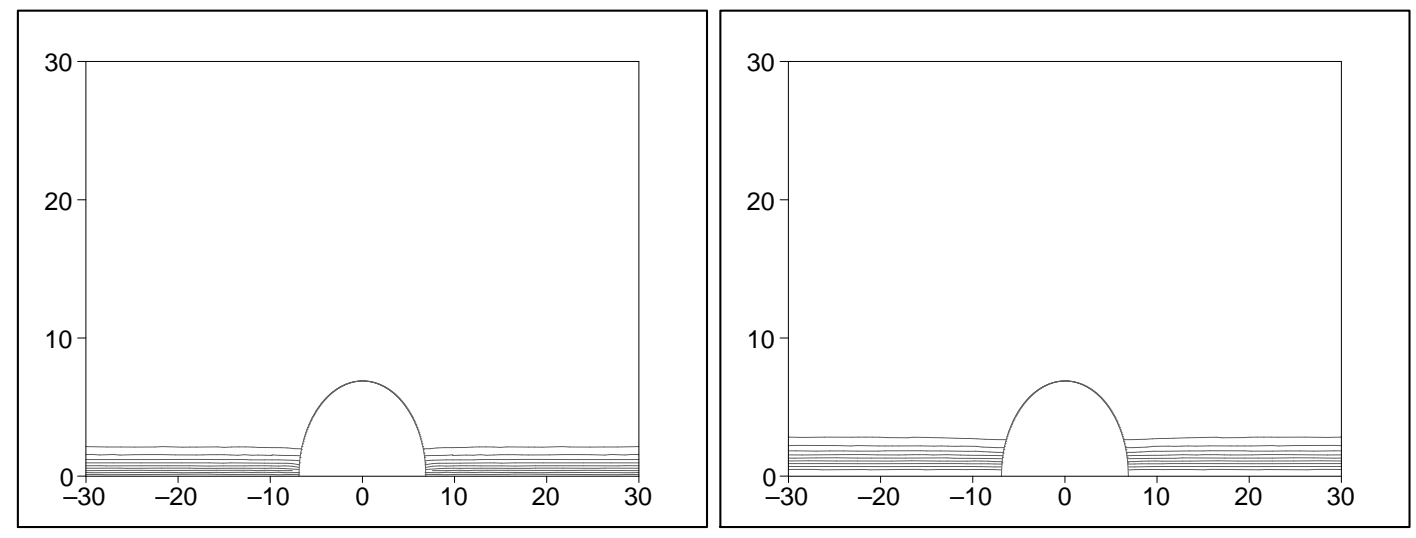

Figure 1: $X$ and $P$ contours ( $X$ and $P$ increase from 0.1 to 0.9 upward and downward respectively) in the background of Kerr-AdS black hole with $l=5$ and $a=0$. The mass of black hole is equal to 10 and horizon is located in $r_{H}=6.89$.

[5] P. Cruschiel, gr-qc/9402032 (unpublished).

[6] A. Chamblin, J.M.A. Ashbourn-Chamblin, R. Emparan, A. Sornborger, Phys. Rev. D58, 124014 (1998).

[7] A. Chamblin, J.M.A. Ashbourn-Chamblin, R. Emparan, A. Sornborger, Phys. Rev. Lett. 80, 4378 (1998).

[8] F. Bonjour, R. Gregory, Phys. Rev. Lett. 81, 5034 (1998).

[9] F. Bonjour, R. Emparan, R. Gregory, Phys. Rev. D59, 84022 (1999).

[10] T. Torii, K. Maeda and M. Narita, Phys. Rev. D59, 064027 (1999).

[11] T. Torii, K. Maeda and M. Narita, Phys. Rev. D64, 044007 (2001).

[12] M.H. Dehghani, A.M. Ghezelbash and R.B. Mann, hep-th/0105134.

[13] M.H. Dehghani, A.M. Ghezelbash and R.B. Mann, Phys. Rev. D65, 044010 (2002).

[14] H.B. Nielsen and P.Olesen, Nucl. Phys. B61, 45 (1973).

[15] W. H. Press, S. A. Teukolsky, W. T. Vetterling and B. P. Flannery, "Numerical Recipes in FORTRAN", Cambridge University Press (1992).

[16] D. Kramers, H. Stephani, E. Herlt and M. MacCallum, "Exact Solutions of Einstein's field equations", Cambridge University Press (1980). 

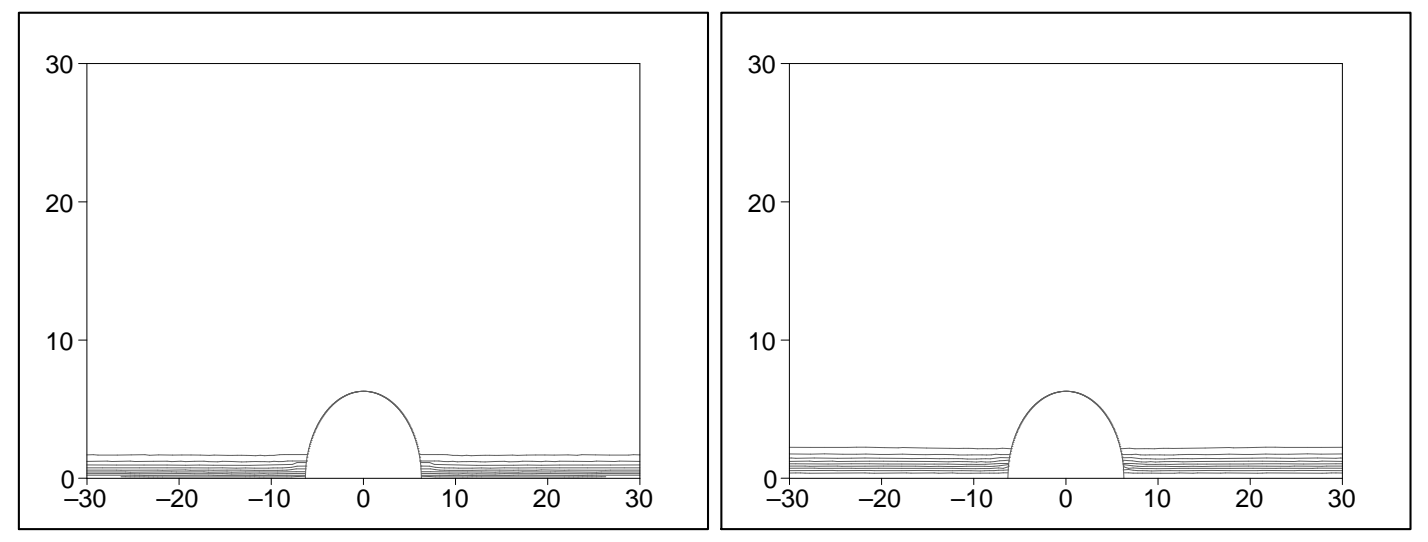

Figure 2: $X$ and $P$ contours ( $X$ and $P$ increase from 0.1 to 0.9 upward and downward respectively) in the background of Kerr-AdS black hole with $l=5$ and $a=3$. The mass of black hole is equal to 10 and horizon is located in $r_{H}=6.30$.
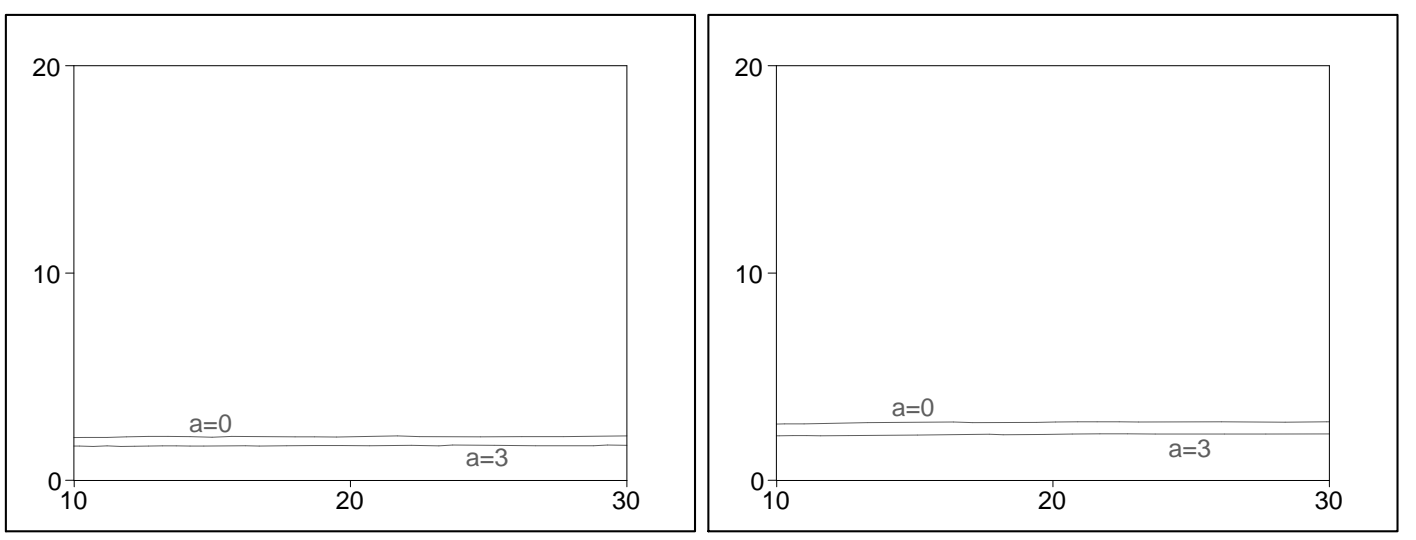

Figure 3: $X=0.9$ and $P=0.1$ contours in the presence of $l=5$ Kerr-AdS black hole for different values of the rotation parameter $a=0,3$. The black hole horizon is located off to the left of the figure at $r_{H}=6.89,6.30$ respectively. 

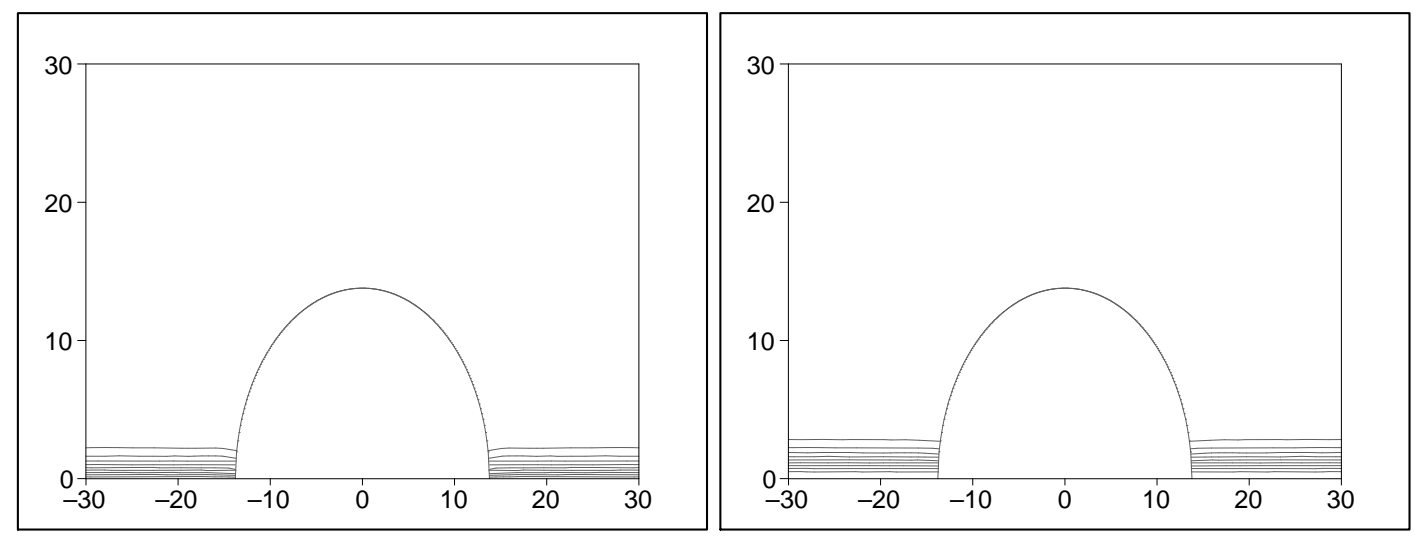

Figure 4: $X$ and $P$ contours ( $X$ and $P$ increase from 0.1 to 0.9 upward and downward respectively) in the background of Kerr-AdS black hole with $l=10$ and $a=0$. The mass of black hole is equal to 20 and horizon is located in $r_{H}=13.79$.
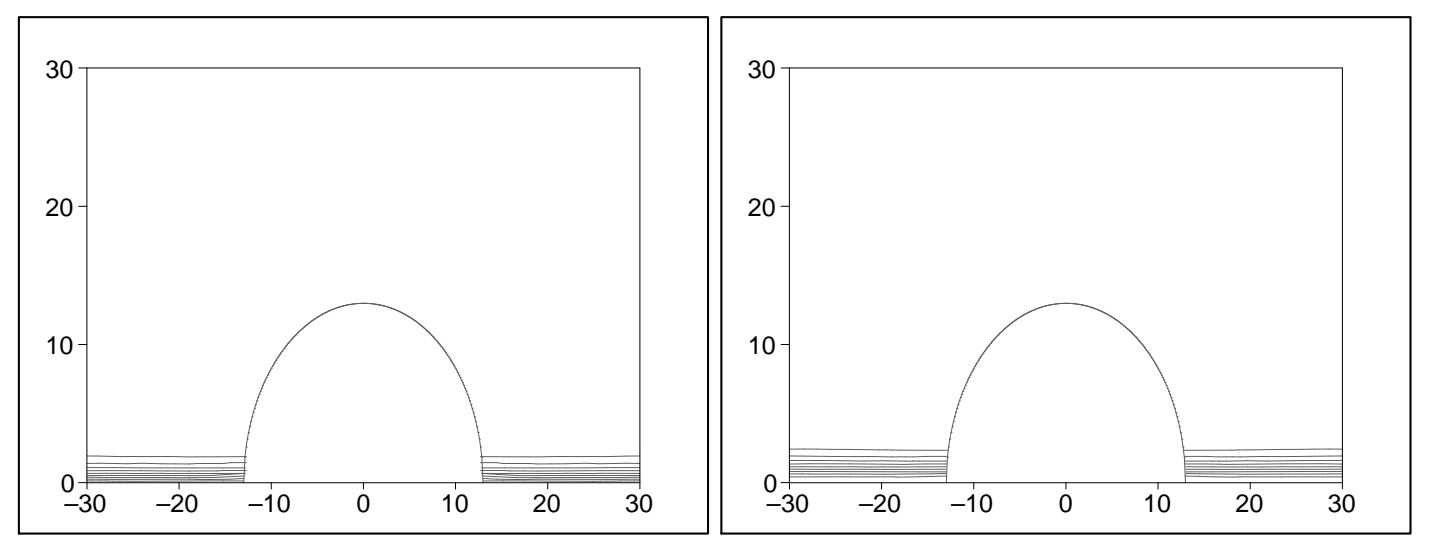

Figure 5: $X$ and $P$ contours ( $X$ and $P$ increase from 0.1 to 0.9 upward and downward respectively) in the background of Kerr-AdS black hole with $l=10$ and $a=5$. The mass of black hole is equal to 20 and horizon is located in $r_{H}=12.98$. 

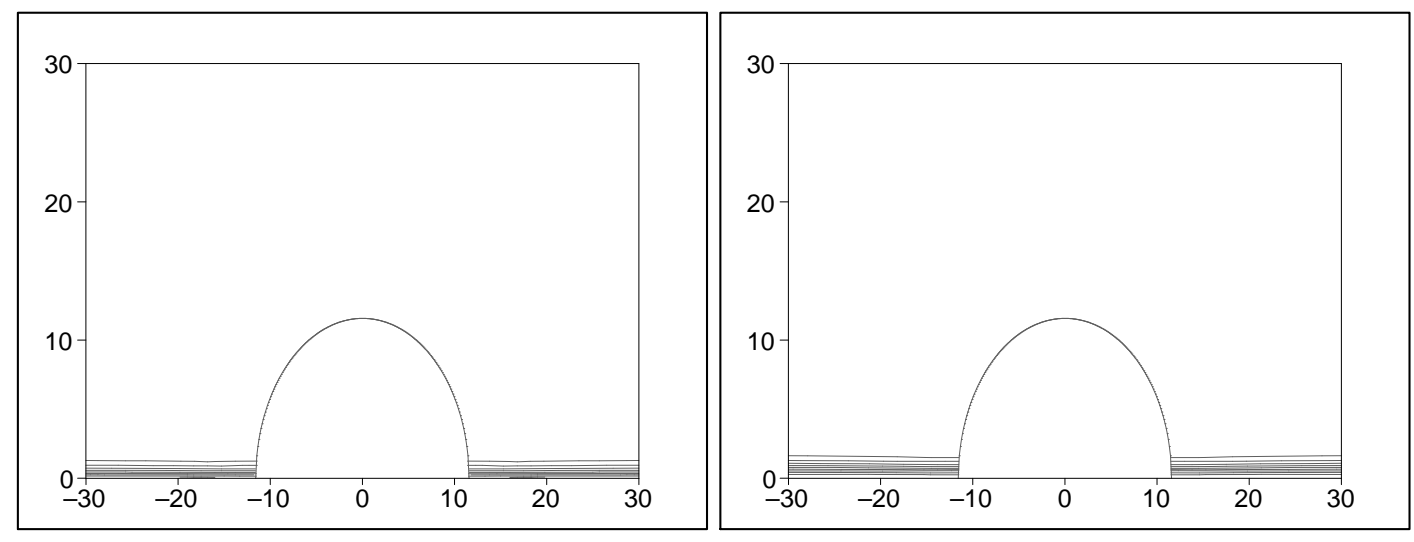

Figure 6: $X$ and $P$ contours ( $X$ and $P$ increase from 0.1 to 0.9 upward and downward respectively) in the background of Kerr-AdS black hole with $l=10$ and $a=8$. The mass of black hole is equal to 20 and horizon is located in $r_{H}=11.57$.
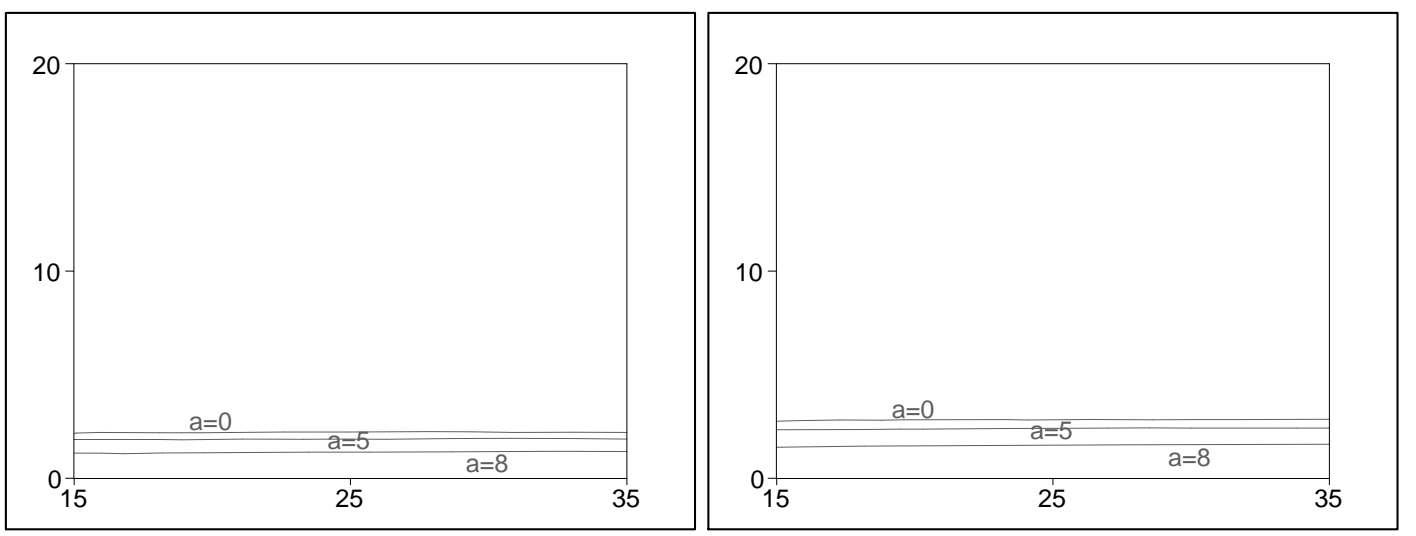

Figure 7: $X=0.9$ and $P=0.1$ contours in the presence of $l=10$ Kerr-AdS black hole for different values of the rotation parameter $a=0,5,8$. The black hole horizon is located off to the left of the figure at $r_{H}=13.79,12.98,11.57$ respectively. 

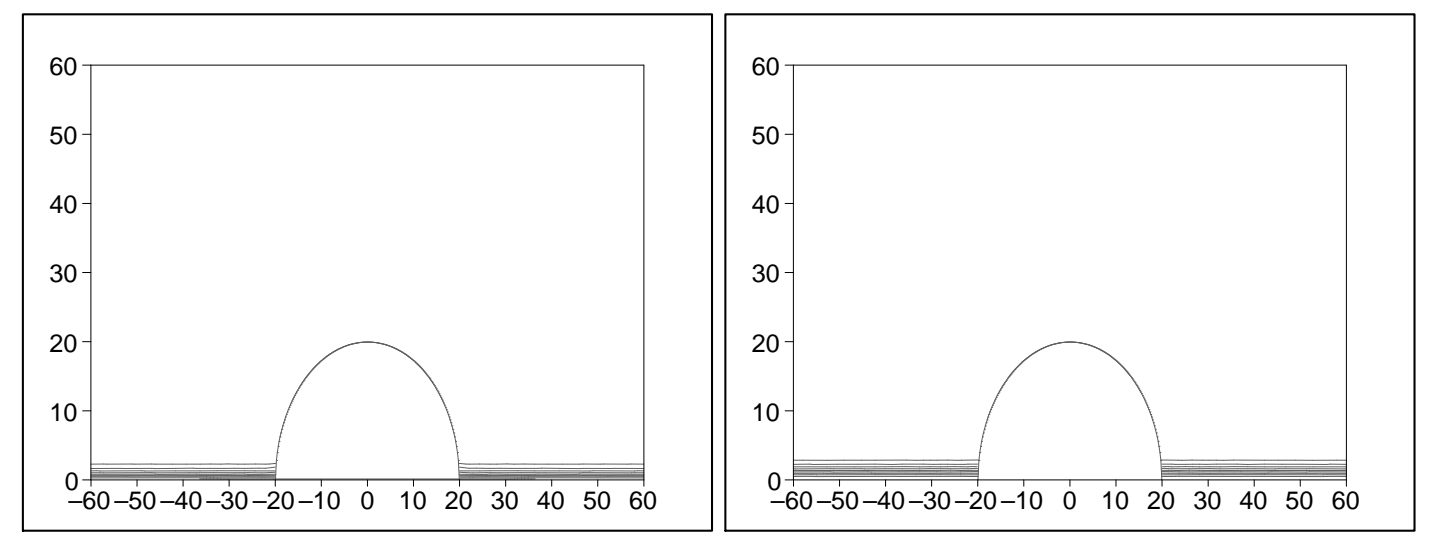

Figure 8: $X$ and $P$ contours ( $X$ and $P$ increase from 0.1 to 0.9 upward and downward respectively) for a string in the presence of Schwarzschild black hole as a limiting case of a Kerr black hole where $a=0$. The mass of the black hole is equal to 10 and horizon is located in $r_{H}=20$.
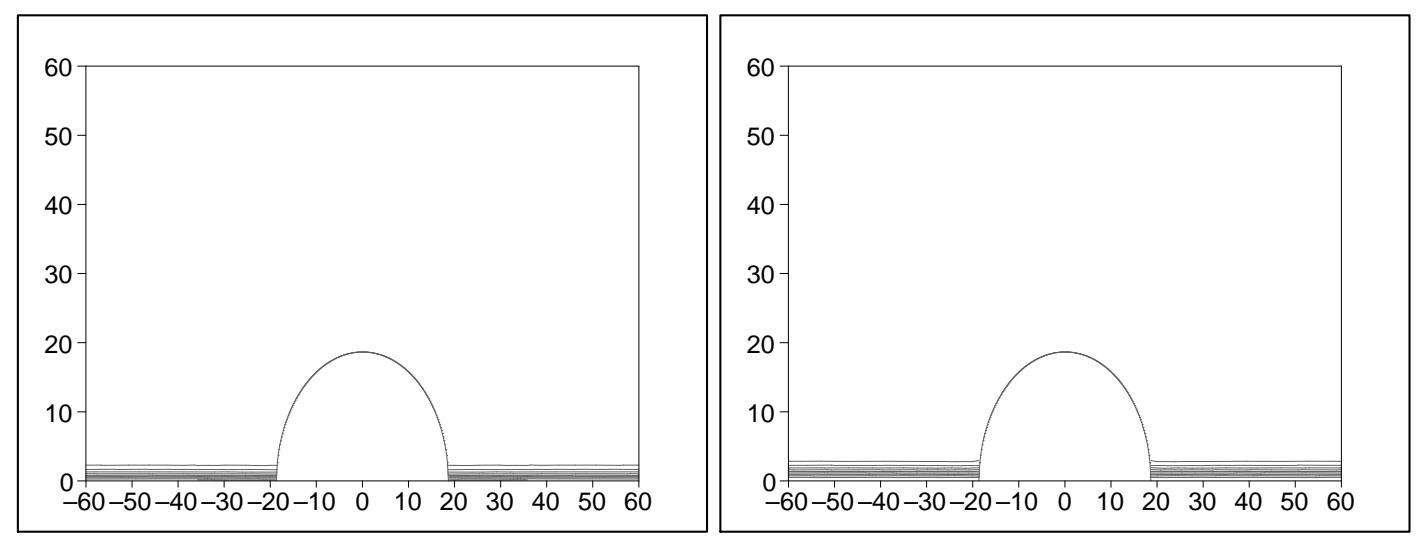

Figure 9: $X$ and $P$ contours ( $X$ and $P$ increase from 0.1 to 0.9 upward and downward respectively) for a string in the presence of Kerr black hole. The mass of black hole is equal to 10, its rotation parameter is equal to 5 and horizon is located in $r_{H}=18.66$. 

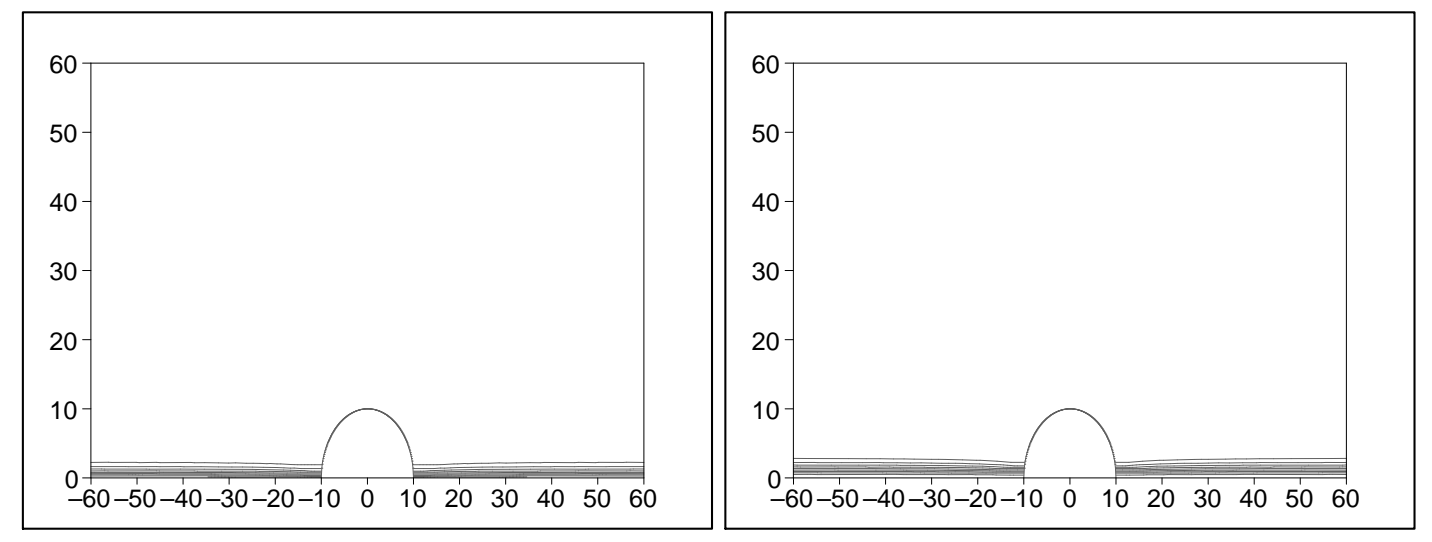

Figure 10: $X$ and $P$ contours ( $X$ and $P$ increase from 0.1 to 0.9 upward and downward respectively) for a string in the presence of extremal Kerr black hole. The mass of black hole is equal to 10 , its rotation parameter is equal to 10 and horizon is located in $r_{H}=10.0$.
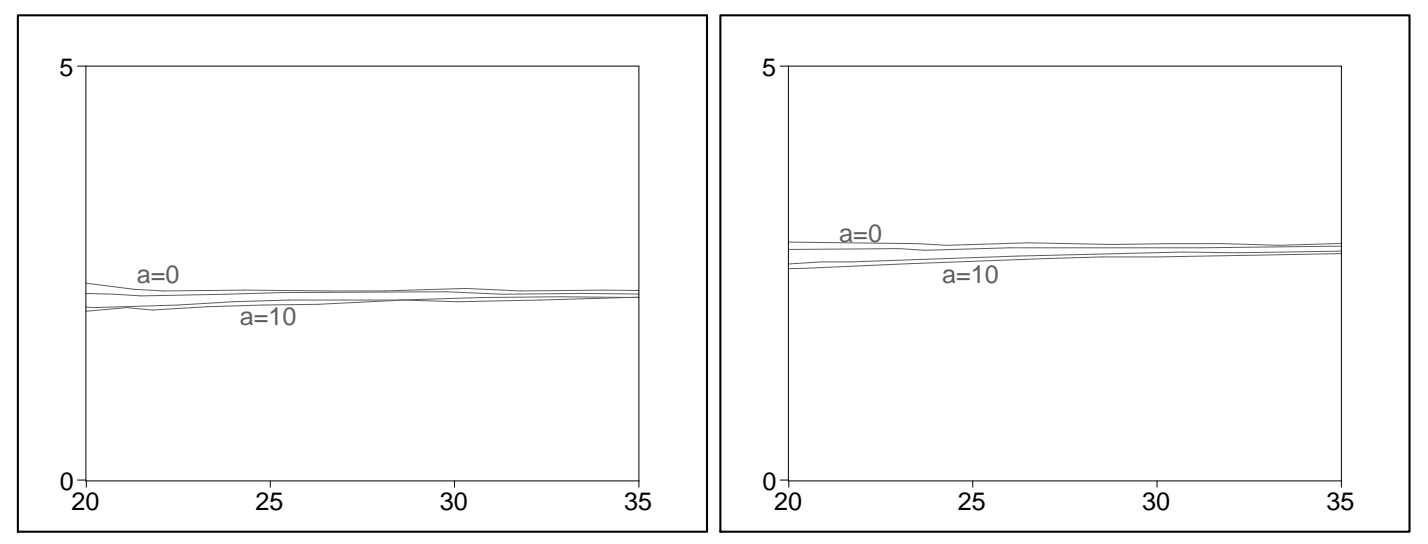

Figure 11: $X=0.9$ and $P=0.1$ contours in the Kerr black hole background of mass $m=10$ for different values of the rotation parameter $a=0,5,9$ and 10 . The black hole horizon is located off to the left of the figure at $r_{H}=20,18.66,14.36$ and 10 respectively. 

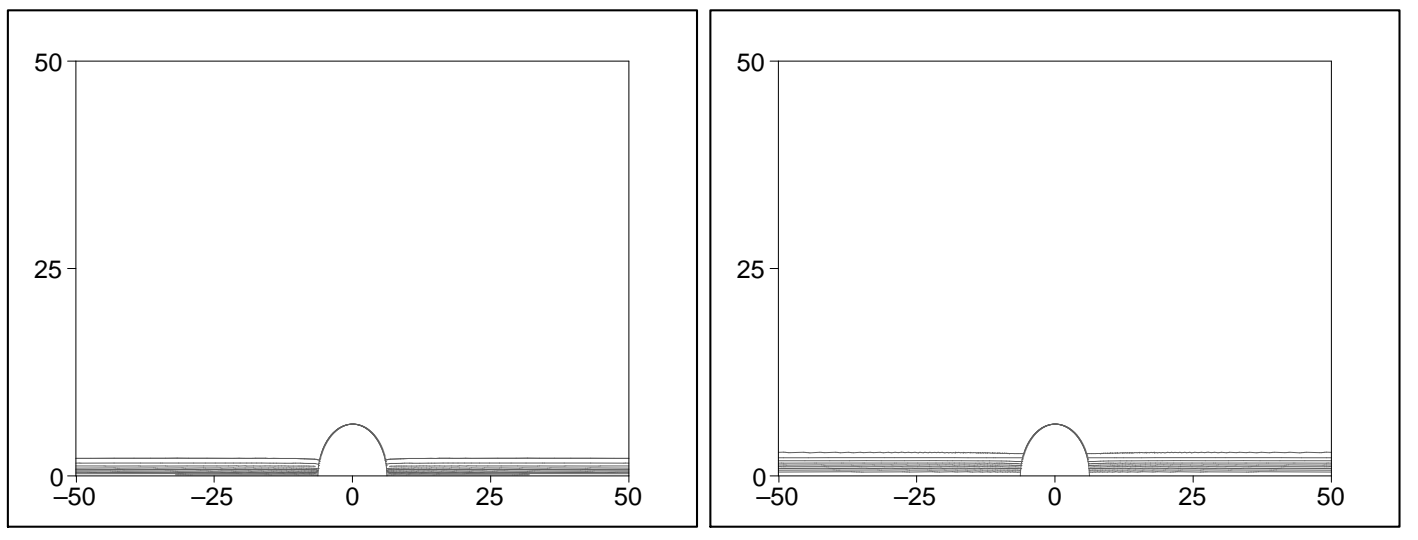

Figure 12: $X$ and $P$ contours ( $X$ and $P$ increase from 0.1 to 0.9 upward and downward respectively) for a string in the presence of Reissner-Nordstrom-AdS black hole with $l=5$. The mass of black hole is equal to 10 , its charge parameter is equal to 5 and horizon is located in $r_{H}=6.25$.
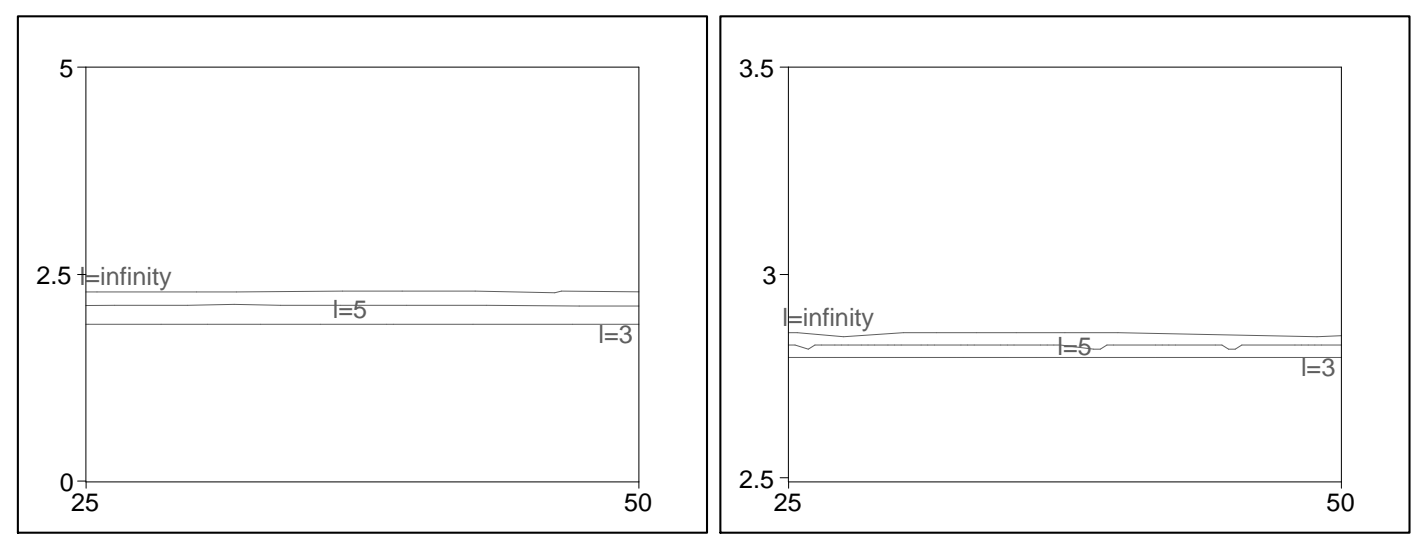

Figure 13: $X=0.9$ and $P=0.1$ contours in the Reissner-Nordstrom-AdS black hole background of mass $m=10$ and charge $Q=5$ for different values of the cosmological constant $l=3,5, \infty$. The black hole horizon is located off to the left of the figure at $r_{H}=4.47,6.25,18.66$ respectively. 

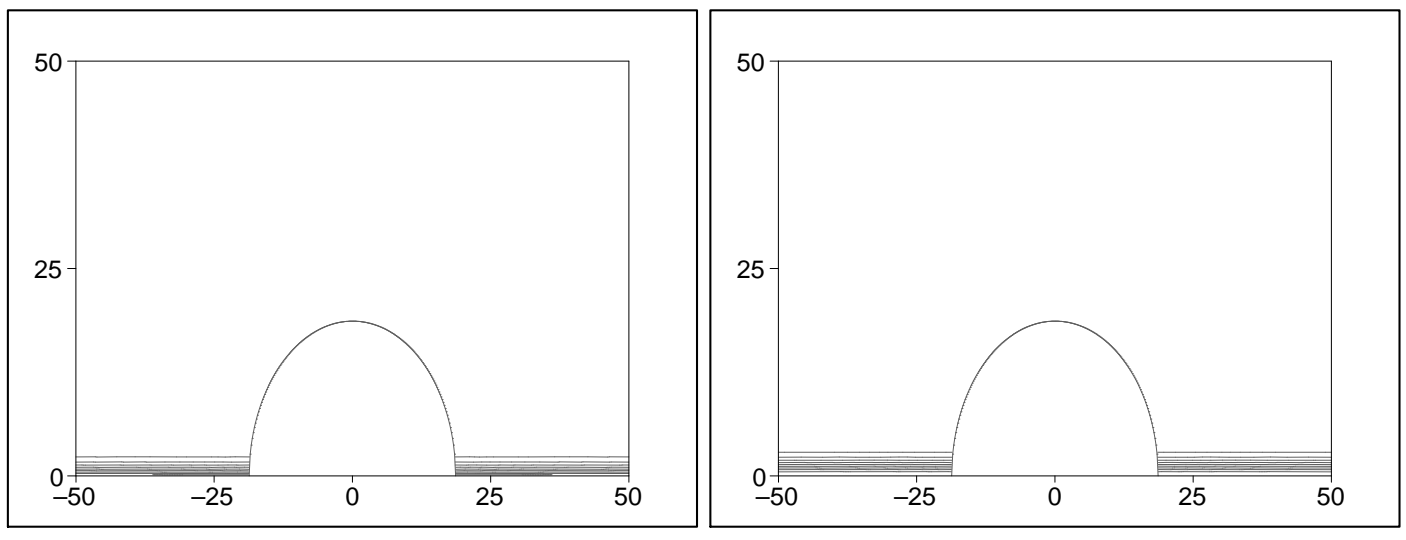

Figure 14: $X$ and $P$ contours ( $X$ and $P$ increase from 0.1 to 0.9 upward and downward respectively) for a string in the presence of Reissner-Nordstrom black hole. The mass of black hole is equal to 10 , its charge is equal to 5 and horizon is located in $r_{H}=18.66$.
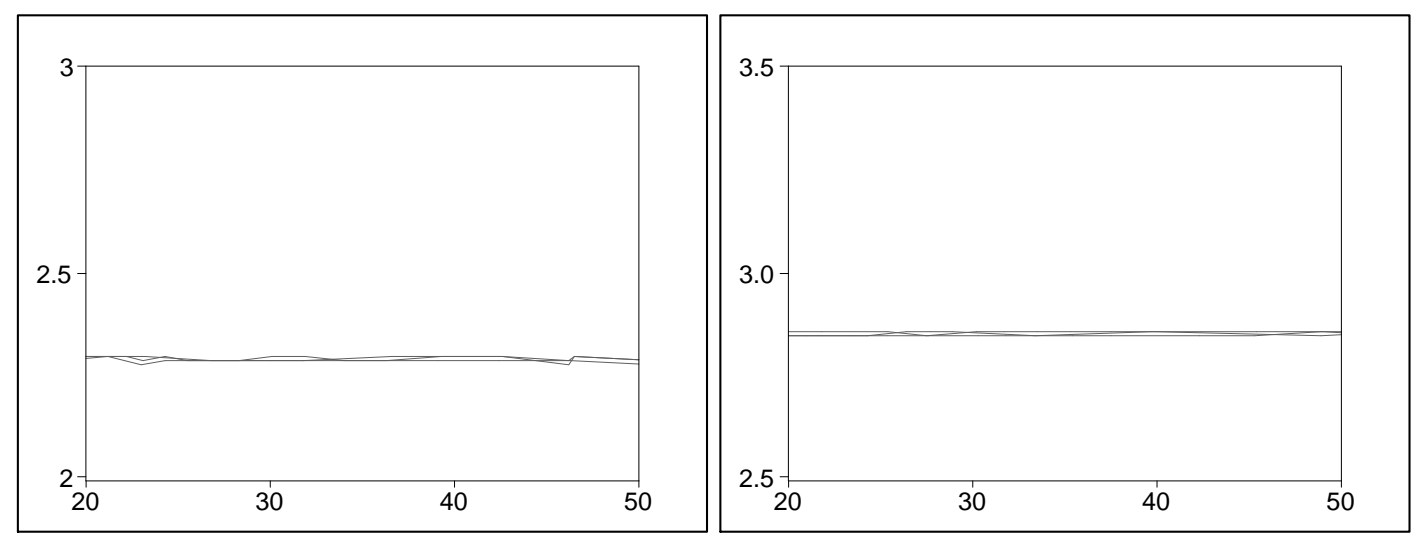

Figure 15: $X=0.9$ and $P=0.1$ contours in the Reissner-Nordstrom black hole background of mass $m=10$ for different values of the charge $Q=0,5,10$. The black hole horizon is located off to the left of the figure at $r_{H}=20,18.66,10$ respectively. 

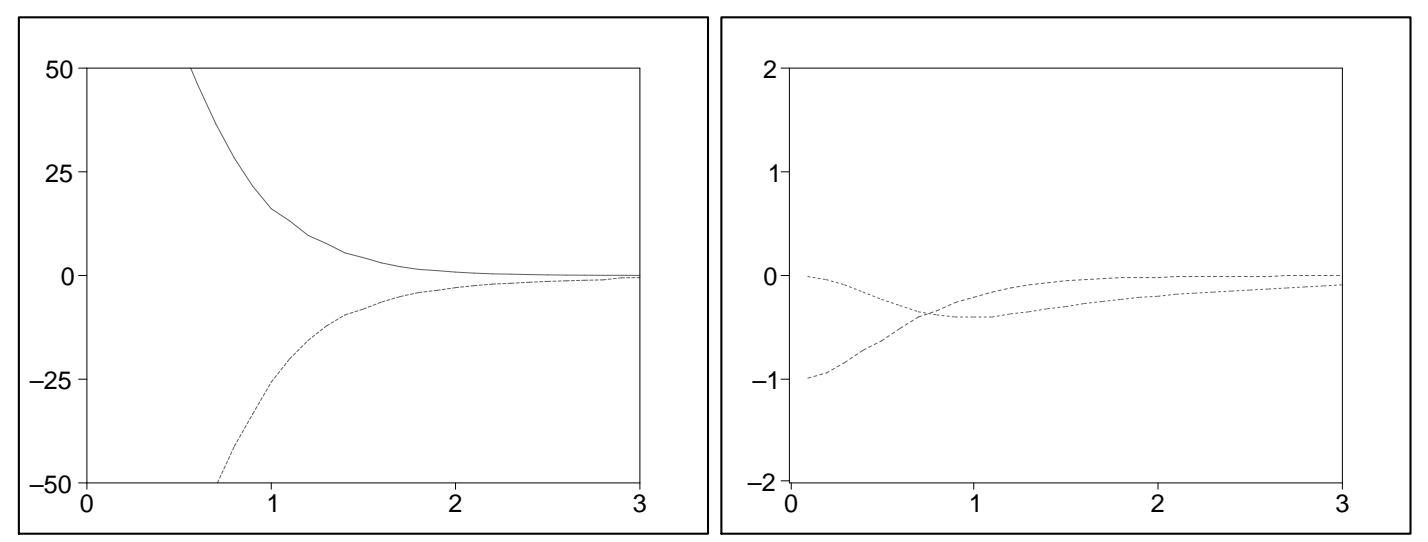

Figure 16: $T_{t t}$ (solid), $T_{\theta \theta}$ (dashed), $T_{\varphi \varphi}$ (dotted) and $T_{r r}$ (dot-dashed) curves versus $\rho$ in $z=8$ in the Kerr-AdS black hole background with $l=5$. Winding number is $N=1$. 